\title{
Gradhiva
}

GRADHIV

Revue d'anthropologie et d'histoire des arts

$24 \mid 2016$

ARTchives

\section{La femme de leurs rêves : Cornelia Schleime et les archives de la Stasi}

Being the woman they wanted her to be: Cornelia Schleime performs her Stasi file

\section{Sara Blaylock}

Traducteur : Camille Joseph

\section{Q OpenEdition}

\section{Journals}

Édition électronique

URL : http://journals.openedition.org/gradhiva/3244

DOI : $10.4000 /$ gradhiva.3244

ISSN : 1760-849X

Éditeur

Musée du quai Branly Jacques Chirac

Édition imprimée

Date de publication : 7 décembre 2016

Pagination : 18-49

ISBN : 978-2-35744-094-4

ISSN : 0764-8928

\section{Référence électronique}

Sara Blaylock, «La femme de leurs rêves : Cornelia Schleime et les archives de la Stasi », Gradhiva [En ligne], 24 | 2016, mis en ligne le 07 décembre 2019, consulté le 19 avril 2019. URL : http://

journals.openedition.org/gradhiva/3244; DOI : 10.4000/gradhiva.3244 


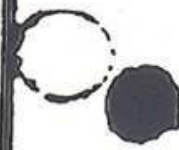

Dem ABV mittlungabericht vom 24.10.83 (Blatt gehenden Informationen oder Hinwei sich die Sch. äußerst unauffäl?

1)
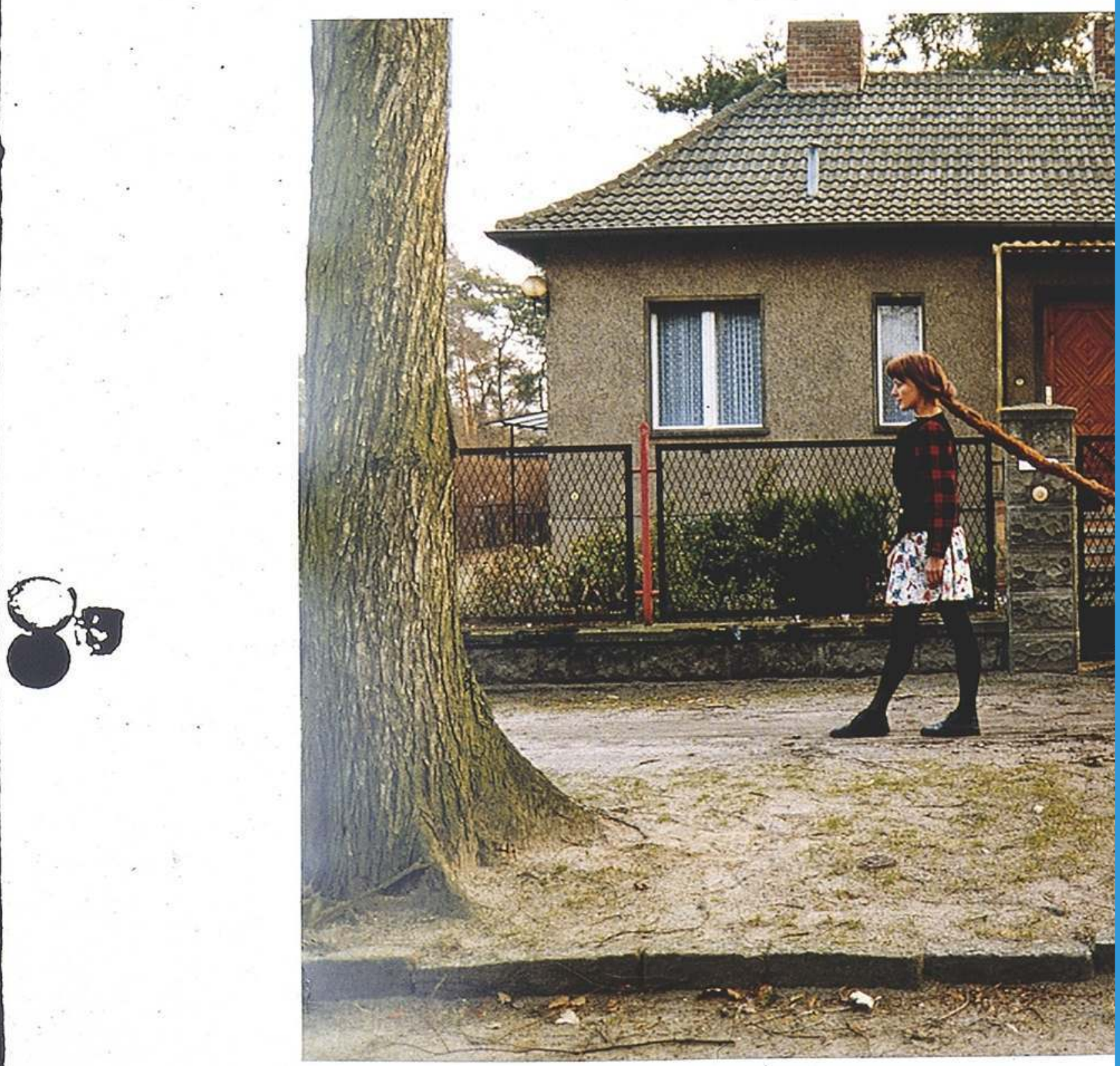

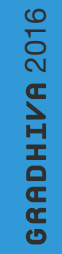




\section{La femme de leurs rêves:}

Cornelia Schleime et les archives de la Stasi

par Sara Blaylock

Il existe aujourd'hui cent onze kilomètres

d'archives de la Stasi désormais ouvertes au public. Cet article examine le travail que l'artiste Cornelia Schleime a réalisé à partir de son dossier de surveillance pour sa série photographique de 1993, Série Stasi.

Peintre reconnue aujourd'hui en Allemagne et figure centrale de la scène artistique expérimentale de RDA dans les années 1980, elle était alors la cible de la police secrète. Pour sa Série Stasi, Cornelia Schleime a utilisé quatorze extraits de son dossier qu'elle a associés à autant d'autoportraits. Les images et le texte se répondent, dessinant ainsi une biographie de l'artiste en deux temps (les années 1980 et l'année 1993), fondée sur deux perspectives irréconciliables (celle de la Stasi et la sienne). En recréant sa biographie par une utilisation sélective de son dossier, l'artiste réalise une œuvre selon ses propres termes. Cet article vise à explorer la frontière entre art et archives, en analysant la façon dont cette performance-archive révèle autant les limites de la source utilisée par l'artiste que le point de vue unique qui est le sien, à la fois en tant que sujet et interprète. 


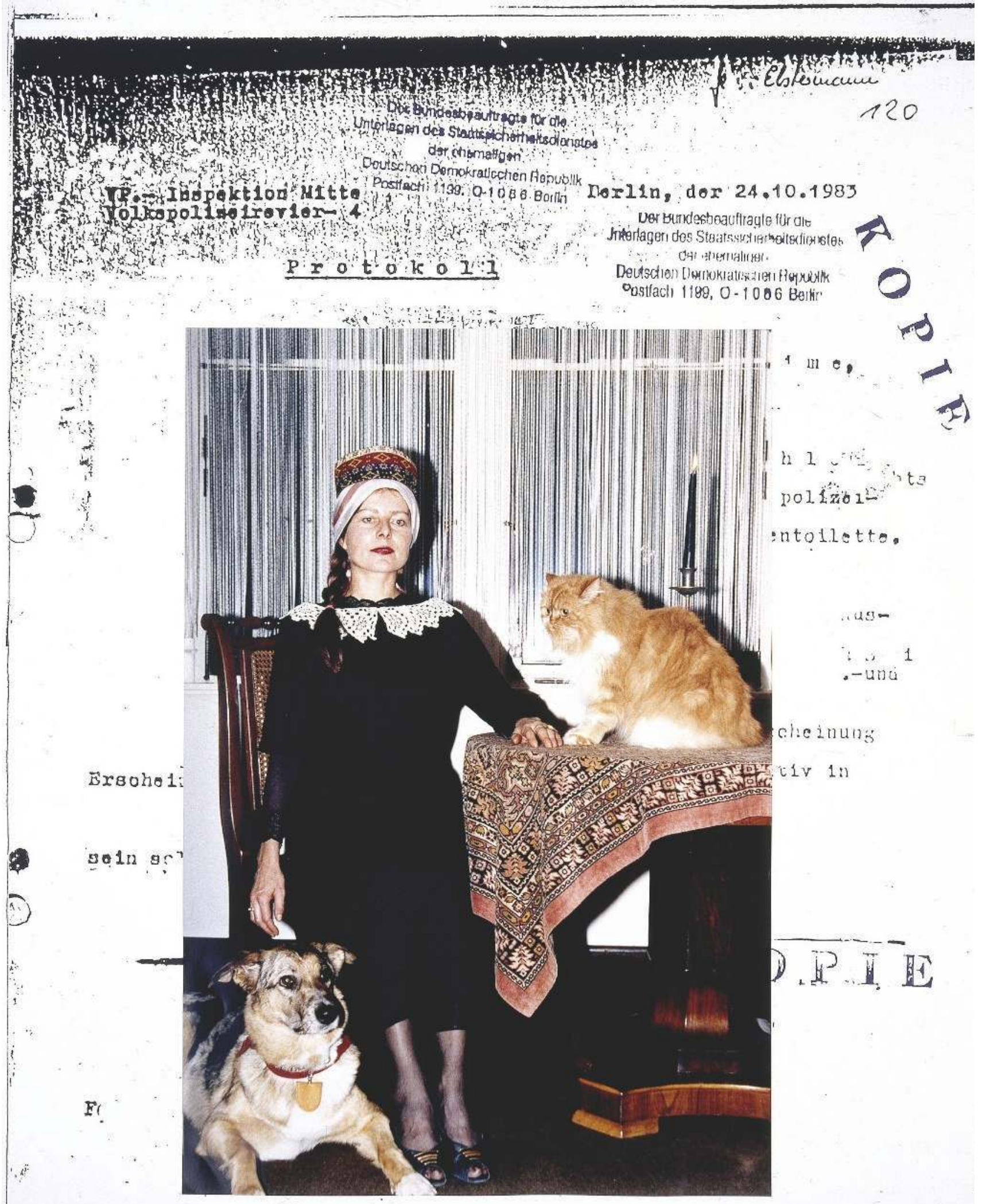

D1. Mobnuag 1ot notduret1g m1t alton Lubolstuoken, was modern dricon aoll; olngorlobtet.

Ihro Haboro Brsobjlaung lat m1t der Aussattuag dor nolnung in abotlmang gobraoht worden und moliw ebenfalls sohr nodern aue- 
Une femme fixe d'un regard absent un point hors champ. Assise bien droite, elle semble tendue comme si elle finissait de prendre une grande inspiration (fig. 1). La table et la chaise sont d'un bois foncé. Sur la table, un tissu à fleurs rappelle le chapeau qui coiffe les cheveux tressés de la femme, recouverts d'un fichu. Elle porte une robe noire stricte, adoucie par un col blanc au crochet. Sur la table se trouvent un énorme chat roux et une bougie allumée. Un berger allemand est assis aux pieds de la femme, qui porte des bas. Le chien lui aussi regarde au loin; il est sur ses gardes, comme s'il allait se mettre à grogner ou à aboyer. De fins rideaux blancs tamisent la lumière du dehors qui éclaire cette scène d'intérieur.

La photographie, un autoportrait de Cornelia Schleime, est placée sur un extrait du dossier de la Stasi, compilé pendant plus de six années d'espionnage de l'artiste par la RDA. En guise de légende figurent ces notes datées du 24 octobre 1983: «L'appartement contient le strict minimum, seuls quelques meubles d'occasion, qui ont pour but de lui donner un air moderne. Son apparence extérieure [celle de Cornelia Schleime] correspond bien à l'aménagement de l'appartement et est elle aussi "censée" faire très moderne. " L'autoportrait, réalisé dix ans plus tard, prend le contrepied ironique du ton railleur et hostile du texte. Plutôt que de verser dans le shabby chic, Cornelia Schleime se déguise sous les traits d'une Allemande austère habitant un intérieur bien soigné. Elle joue ainsi à la femme que la Stasi voulait qu'elle soit. L'image n'en capture pas moins un moment de tension. Les figurants de la scène sont calmes, mais en déséquilibre: le gros chat est installé dangereusement près de la bougie, que le chien, à l'attitude incertaine, pourrait bientôt faire tomber. Et on ne sait pas si, au moment de l'expiration, la tension se relâchera ou sera au contraire à son comble.

\section{L'archive, l'État et l'artiste}

Il existe aujourd'hui quelque cent onze kilomètres d'archives de la Stasi ouvertes au public. Cet article examine le travail que l'artiste Cornelia Schleime a réalisé à partir des centaines de pages de son dossier pour sa série photographique de 1993, Bis auf weitere gute Zusammenarbeit, Nr. 7284/85 (Dans l'attente de la poursuite d'une bonne collaboration, Nr. 7284/85), dite aussi Série Stasi. Peintre reconnue aujourd'hui en Allemagne, Cornelia Schleime était dans les années 1980 une figure centrale de la scène artistique expérimentale de RDA, en tant que peintre, réalisatrice, mais aussi musicienne punk. Elle était alors la cible de la police secrète.

Pour sa Série Stasi, Cornelia Schleime a utilisé quatorze extraits de son dossier qu'elle a associés à autant d'autoportraits. Ce projet, réalisé en 1992 et 1993, quelques mois après que les dossiers de la police secrète de l'ex-RDA furent rendus publics ${ }^{1}$, a servi à l'artiste de catharsis ironique et sarcastique: “C'était une façon pour moi de me libérer de ce traumatisme ${ }^{2}$." Le choc d'avoir à revivre le passé à travers ces documents fut double, à cause de la réalité inconcevable qu'ils révélaient: des dizaines de milliers d'Allemands de l'Est furent des collaborateurs non officiels (Inoffizieller Mitarbeiter, IM) de la Stasi, rédigeant des rapports sur leurs collègues, amis, amants et membres de leur famille (Müller-Enbergs 2008: 3); et ils furent plus nombreux encore, notamment les gérants d'immeuble qui consignaient la vie de leurs
1. Le gouvernement allemand ratifia la première version de la loi sur les archives de la Stasi le 20 décembre 1991.

2. Entretien téléphonique avec Cornelia Schleime, le 25 février 2015. Lorsque, dans la suite du texte, l'artiste est citée sans référence bibliographique, il s'agit d'extraits de ce même entretien. 


\section{ci-contre}

fig. 2

Cornelia Schleime,

Bis auf weitere gute

Zusammenarbeit,

Nr. 7284/85, série

en 15 parties, 1993 photographie sérigraphiée,

$100 \times 70 \mathrm{~cm}$ (C) Cornelia

Schleime, avec l'aimable

autorisation de la galerie

Michael Schultz, Berlin. locataires dans des livres consultés régulièrement par la police et la Stasi, à contribuer ainsi au système complexe de surveillance établi en RDA. Quand Cornelia Schleime eut pour la première fois accès à son dossier en 1992, elle fut obligée, comme tant d'autres, de constater le rôle que certains confidents, alliés ou encore voisins avaient en réalité joué dans ces années qui furent pour beaucoup très difficiles. Impossible de régler les comptes qui ressurgissaient alors du passé - «Tout le monde ne parlait que de ça! »; mais pour l'artiste, il fallait en faire quelque chose de productif. Dans le journal qu'elle tenait au début des années 1990, elle avait écrit: “C'est de constamment regarder en arrière qui me rend le plus malade. Je ne veux pas perdre mon temps. J'ai envie de continuer à travailler à mes nouveaux tableaux, mais je suis dégoûtée. Non seulement ils ont trahi des gens. Mais en plus ils nous volent notre temps. » (Schleime 2010: 75)

Il est communément admis que si la Stasi surveillait les artistes, c'est parce que leurs œuvres étaient potentiellement dissidentes et contestataires. Dans sa Série Stasi, Cornelia Schleime s'attaque aux craintes de la police secrète en présentant sa vie domestique comme signe de dissidence. La sélection qu'elle opère dans son dossier vise à exposer la banalité "perverse " de ces documents, et à pointer la complicité des citoyens allemands au sein d'un système de contrôle corrompu: "Ces rapports étaient si minables, ils faisaient partie d'une culture de la dénonciation dans laquelle les gens balançaient leurs voisins. [...] C'était cela que je voulais montrer. » Ainsi, Cornelia Schleime a volontairement laissé de côté des documents plus politiques, parmi lesquels des rapports sur sa pratique artistique, parce que les observations de sa vie de tous les jours étaient "suffisamment surréalistes et absurdes". Les commentaires figurant dans son dossier vont de jugements portés sur son apparence («moderne et occidentale») jusqu'à l'heure et au type de ses visites masculines («juste pour le plaisir»), en passant par des remarques sur son isolement («elle n'a aucun contact avec ses voisins"). L'artiste combine des autoportraits instantanés aux documents de surveillance pour mettre en lumière le rôle de la vie privée aux yeux d'un État qui évaluait de cette façon la menace que représentaient certains citoyens pour sa souveraineté. Elle pouvait ainsi faire connaître au monde entier "ce à quoi [elle] étai[t] confrontée en RDA, et ", ajoute-t-elle, "pourquoi tout ce système s'est écroulé».

On peut parler, dans le cas de Cornelia Schleime, d'une «anarchive», un terme que Hal Foster utilise pour décrire le travail des artistes qui réutilisent et interprètent des archives pour soulever des questions spécifiques (Foster 2004: 5). Par son usage rebelle des archives, la Série Stasi de Cornelia Schleime est également une œuvre de «citation performative», un concept théorisé par Judith Butler pour expliquer la manière dont une identité déviante se forme bien souvent en affectant ou en imitant la langue de l'oppresseur. L'accent mis par Judith Butler sur les conditions matérielles produisant la performance du genre est utile pour décrire le rôle que jouent l'imitation, la moquerie, la confrontation, l'exagération et l'ironie dans les photographies de Cornelia Schleime, ainsi que l'usage qu'elle fait du texte lui-même.

Le cadre théorique proposé par Hal Foster et Judith Butler constitue un point central dans l'analyse développée ici, qui vise à contextualiser la Série 


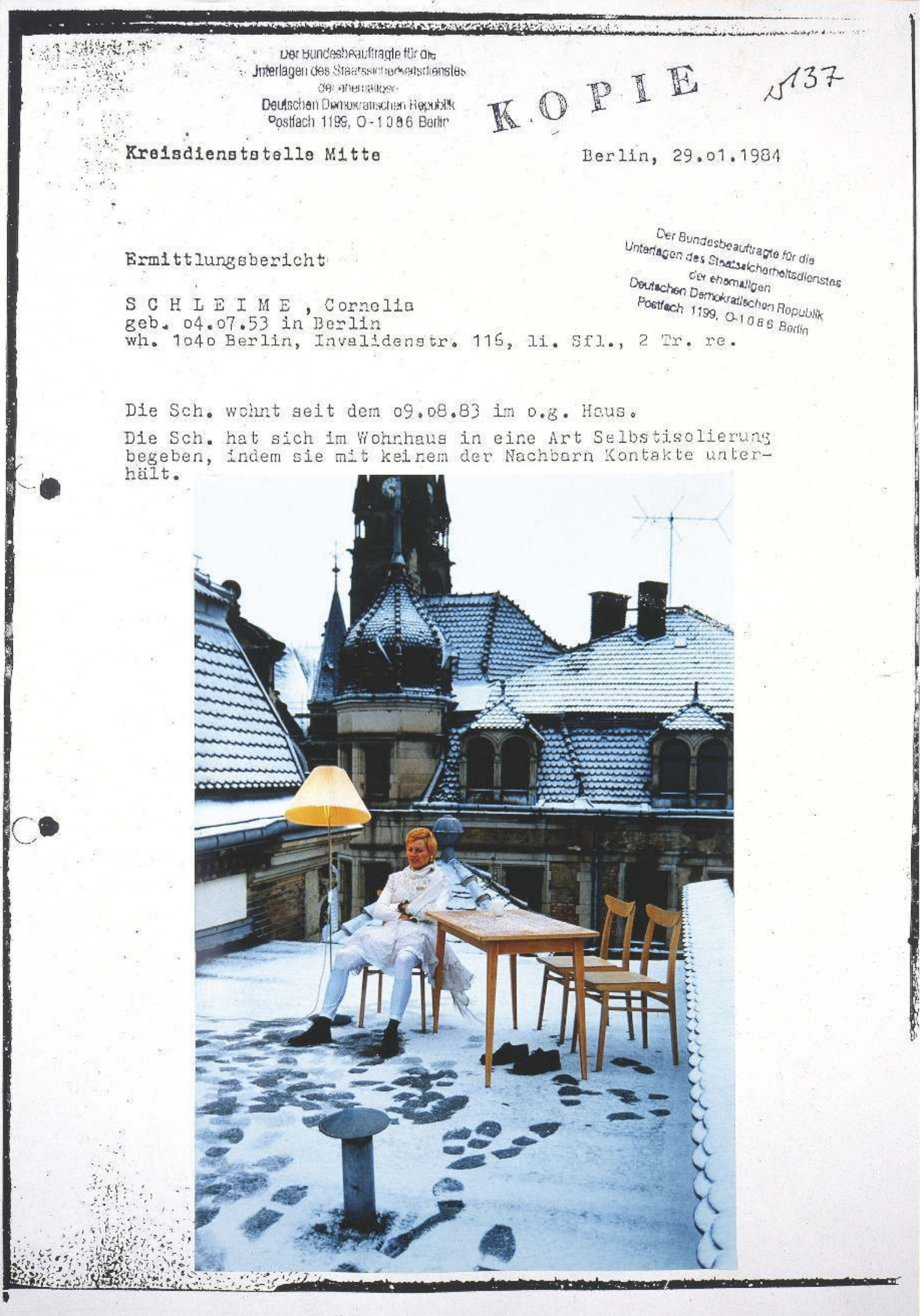


Stasi de Cornelia Schleime de trois façons. Dans un premier temps, on s'interroge sur ce que ce projet artistique dit du système de surveillance de la RDA. L'œuvre est dans un second temps examinée à la lumière des théories de l'archive pour comprendre ce que signifie le fait de s'emparer de ces documents pour critiquer l'appareil étatique. Enfin, il s'agit de comparer ce projet à d'autres œuvres convoquant elles aussi des archives ou s'intéressant éga lement à la question du genre et de la surveillance; cela donnera l'occasion de nuancer l'influence du contexte politique sur les pratiques artistiques. La Série Stasi fait partie des premières œuvres à utiliser les dossiers de la police secrète de RDA. Une analyse du point de vue de l'art contemporain, qui permettrait en particulier de sortir du cadre de la guerre froide, n'a pas encore été proposée pour cette œuvre, qui fait toutefois l'objet d'une mention importante dans quelques catalogues d'exposition (voir Kaiser et Petzold 1997; Gillen 2002; Pejić 2009). Aucune étude ne s'est encore attachée à replacer ce travail dans le contexte des pratiques artistiques contemporaines utilisant des archives ou traitant de la surveillance; pourtant la Série Stasi interroge elle aussi l'information et le pouvoir, la nation et l'archive, la mémoire et le document, l'espionnage et ses conséquences matérielles, ainsi que les formes alternatives de représentation de soi. sur un toit enneigé, aménagé comme une salle à manger (fig. 2). Vêtue d'une robe blanche et de collants blancs eux aussi, enfoncés dans des bottines noires, elle est avachie sur une chaise en bois. Son attitude est à la fois anticonformiste et contemplative. Elle a le regard rivé au sol à quelques mètres d'elle, là où des empreintes de pas mènent à une paire de chaussures placée sous la table. Il y a deux chaises identiques bien rangées d'un côté de la table. Les chaussures sont très exactement mises là où on s'attendrait à trouver deux pieds. À la droite de Cornelia Schleime, une lampe diffuse une lumière jaune. Tout autour d'elle, à l'arrière-plan, se dressent des bâtiments richement décorés. Une tour d'horloge s'élève au loin.

Au-dessus de la photographie, le texte de la Stasi confère à la scène une atmosphère de misère et d'esseulement: "La Sch. vit dans la maison mentionnée plus haut depuis le 9 août 1983. La Sch. pratique une sorte d'isolement, elle n'entretient aucun contact avec ses voisins. " Cette référence à «la Schleime» renvoie à un faux-semblant d'objectivité. Le rapport de la Stasi est censé produire des données et non des jugements personnels. Or Cornelia Schleime se représente comme une personne difficile à classer: c'est quelqu'un qui vit sur un toit plutôt que dans une maison, qui a des compagnons invisibles, et qui s'habille de manière plus ostentatoire que fonctionnelle. Bien que l'artiste ne remette pas en question les impressions de l'observateur, la mise en scène surréaliste de son autoportrait est ambiguë. Elle traite le texte comme une approximation biographique, le document semblant s'accorder momentanément avec sa mémoire des faits. Mais il n'existe pas de correspondance exacte entre le document et le souvenir, il y a au contraire une ambivalence d'un point de vue temporel. Car est-ce le passé qui est reflété ici? Et si oui, selon la perspective de qui? Ou bien est-ce la relation actuelle de Cornelia Schleime avec son passé qui est représentée? 


\section{L'impulsion anarchivistique}

L'art archivistique est autant préproduction qu'il est postproduction: ces artistes, qui s'intéressent moins aux origines absolues qu'aux traces obscures (peut-être l'expression "impulsion anarchivistique" seraitelle plus appropriée), sont souvent attirés par des débuts manqués ou des projets inachevés - dans l'art ou dans l'histoire - qui pourraient offrir de nouveaux points de départ.

(Foster 2004: 5)

En ajoutant le préfixe «an-», Foster insiste ici sur le caractère anarchique et, plus loin, anomique (c'est-à-dire affranchi de toutes règles) des pratiques des artistes travaillant avec des archives. Dans son texte de 2004, "An Archival Impulse», il examine le travail de Thomas Hirschhorn, Tacita Dean et Sam Durant, qui se détournent du cynisme postmoderne des dernières décennies pour aller vers plus d'agentivité et de sélectivité dans la collecte et la présentation de l'information. Chacun de ces artistes assemble une sorte de facticité circonscrite ou subjective en combinant des documents d'archives de manière dialectique. Hirschhorn et Dean produisent des œuvres où se mêlent et s'unissent à plaisir muses personnelles et fantasmes; Durant quant à lui choisit de faire ce qu'il appelle de «mauvaises combinaisons" (cité dans Foster 2004: 20), c'est-à-dire de produire de la friction iconographique et symbolique. Ce sont là des moyens de retourner les réalités matérielles contre le formalisme détaché de l'art conceptuel. Le travail de ces artistes est anarchique en ce qu'il préfère la trace à la totalité. Il est anomique dans le sens où les traces sélectionnées pour représenter l'archive sont personnelles, et même délibérément idiosyncratiques. Foster écrit que ce genre de travail «prend le parti de la fragmentation anomique non seulement comme condition de représentation mais de libération, et propose à cette fin de nouveaux ordres d'association affective, quoique partiels et provisoires, y compris quand il capte la difficulté, et parfois l'absurdité, d'une telle posture" (ibid.: 21). L'absurdité de l'anarchive est incontrôlée, elle n'est pas planifiée, elle est même antisociale. De la même manière, le travail de Cornelia Schleime offre lui aussi une vision partielle et sélective des archives de la Stasi. L'hyperbole de ses photographies met à nu la fiction du projet, qui n'est elle-même qu'un miroir de la fiction à l'œuvre dans les dossiers de surveillance. Notre accès à ces textes dépend entièrement du désir de l'artiste. C'est elle qui décide quels éléments de son dossier elle va utiliser et comment elle va les utiliser. Elle orchestre les conditions de notre rencontre avec une histoire de la surveillance, même si elle consent à montrer des documents biographiques qu'elle n'a pas ellemême créés. En faisant ainsi un usage «anarchivistique» (anarchival) de son dossier, Cornelia Schleime réalise une performance de la sélectivité de l'appareil de surveillance de la RDA, qui cherchait à la contenir, à la classer et à la décrire. Cette relation au dossier, prudente et spontanée, oblige le spectateur, comme dans le cas de Durant, à reconsidérer son impression immédiate de ce que cette archive - une manifestation du pouvoir étatique - peut ou doit dire du passé.

L'approche anarchivistique peut se dissimuler derrière une attention à la forme, qui tout à la fois masque les interventions de l'artiste sur l'archive et attire l'attention sur celle-ci. La Série Stasi contient quatorze images et 


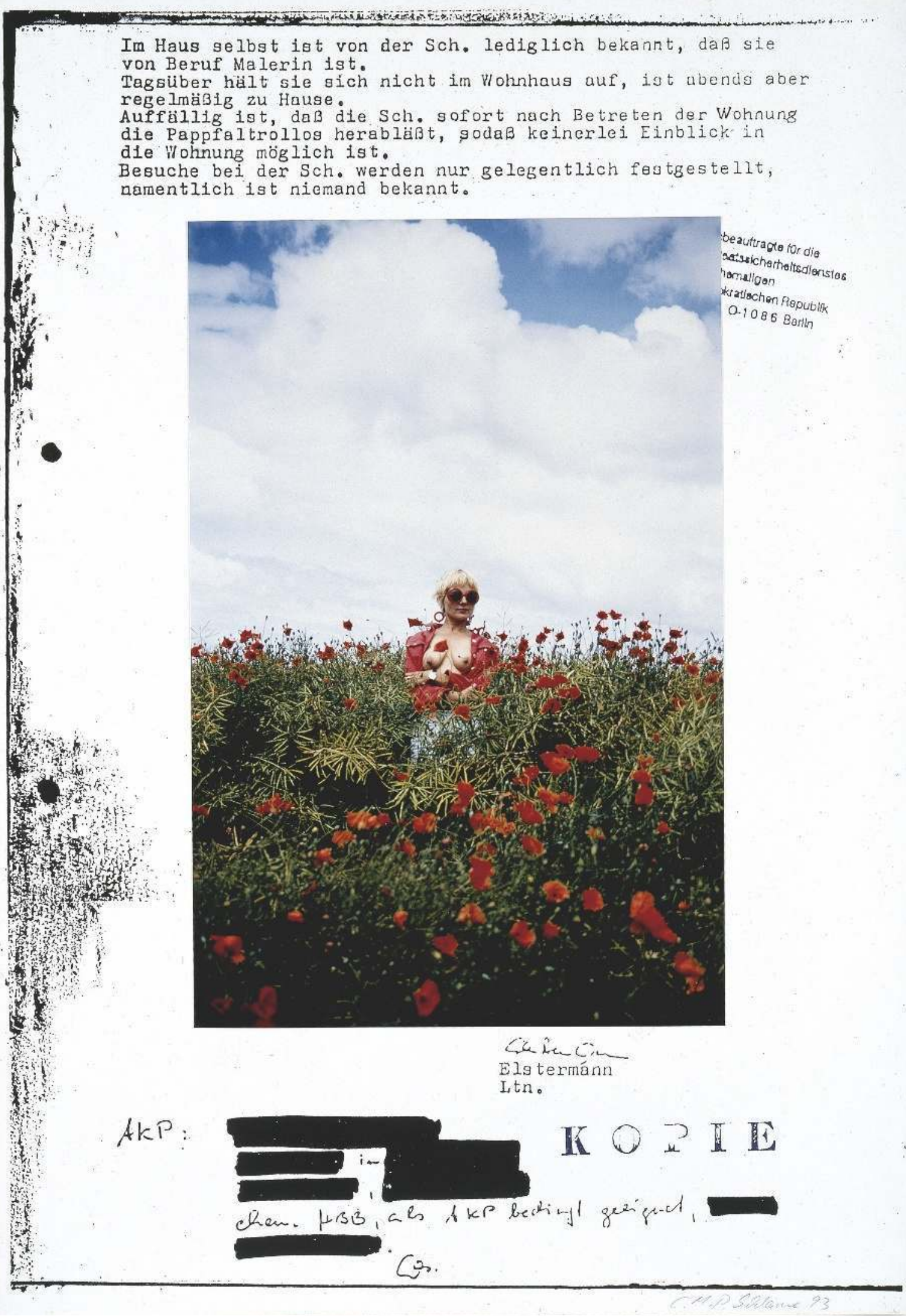


documents écrits associés par paires, quatorze planches de même dimension (fig. 4). À chaque exposition, l'ordre de la série peut varier. À deux reprises Cornelia Schleime a associé plus d'une photographie avec un seul morceau de texte ${ }^{3}$. Elle expliqua lors de notre entretien téléphonique que ce choix était arbitraire. Cela nous invite à prendre en compte la malléabilité de l'histoire, c'est-à-dire les soubassements idéologiques et psychologiques cachés dans l'archive (Foucault 1969; Derrida 1995). La taille de la planche comme celle du panneau d'assemblage - est toujours la même: $100 \times 70 \mathrm{~cm}$. Ces impressions multiplient par trois la taille de la page originale extraite des archives. Le document A4 devient l'œuvre B1. Les photographies, pour la plupart en couleurs, occupent un tiers de la page et sont plus ou moins centrées. Elles sont imprimées séparément sur du papier photographique standard, puis collées par-dessus les reproductions sérigraphiées des documents de la Stasi.

Dimensionnées de manière à correspondre à l'archive, les photographies semblent avoir été prises par la police secrète et glissées dans le dossier comme des pièces complémentaires. Sur toutes les photographies, à une exception près, l'artiste est seule; dans un cliché sur deux, elle regarde directement le spectateur; elle porte à chaque fois une tenue différente. Ses cheveux sont souvent coiffés d'une perruque ou d'un chapeau. Elle pose avec des accessoires dans des espaces divers, le plus souvent domestiques. Certaines images sont sexuellement provocantes. L'artiste se représente alors comme une Occidentale et alimente ainsi les jugements portés sur elle par ses observateurs. Dans d'autres portraits, elle feint d'être maternelle. Elle apparaît alors, dans sa tenue pratique, comme une socialiste modèle, prête pour l'action. D'autres images, telle la scène de la salle à manger sur le toit, glissent dans le rêve et l'imaginaire. Chaque photographie est une performance du texte auquel elle est associée. La portée de cette performance, qui est en réalité plutôt une citation, invite le spectateur à spéculer sur ce qui est à la fois présent et manquant sur la page. Tous les textes sont reproduits dans leur version originale, sans traduction.

Les reproductions portent la trace des différentes interventions sur les pages d'origine. De l'encre noire remplit le vide laissé par les trous de la perforatrice. Une bordure noire irrégulière encadre le blanc de la page. Par endroits, des marques de trombone signalent que des documents ont pu être provisoirement attachés ensemble. L'usure ou la qualité médiocre du papier des originaux est rendue par des taches ou des traces inégales, autant d'artefacts produits par la copie carbone, le photocopieur et la sérigraphie. Ce sont là les traces d'usages multiples. Le blanc immaculé du papier d'impression sur lequel l'artiste a reproduit les documents d'archives contraste en effet avec l'état ancien et taché des originaux. II s'agit de représentations de documents conservés dans les archives berlinoises: jaunissant et s'abîmant sous le poids des ans, ils sont glissés parmi les centaines de pages des dossiers numéros 7284 et 7285 . Les textes témoignent de leur passage entre de nombreuses mains depuis leur émission. Des bureaucrates de la Stasi hantent la surface de ces pages de leurs signatures et marginalia. Une seconde bureaucratie se profile, celle des Archives de la Stasi (BStU), qui s'occupe des dossiers depuis le vote de la loi de 1991. Des tampons rectangulaires avec des numéros indiquent la pagination de
3. Ces indications s'appuient sur la monographie de l'artiste (Schleime 2010).

\section{ci-contre et double page suivante}

fig. 3 et 4

Cornelia Schleime, Bis auf weitere gute Zusammenarbeit,

Nr. 7284/85, série en 15 parties, 1993, photographie sérigraphiée, $100 \times 70 \mathrm{~cm}$ @ Cornelia Schleime, avec l'aimable autorisation de la galerie Michael Schultz, Berlin. 

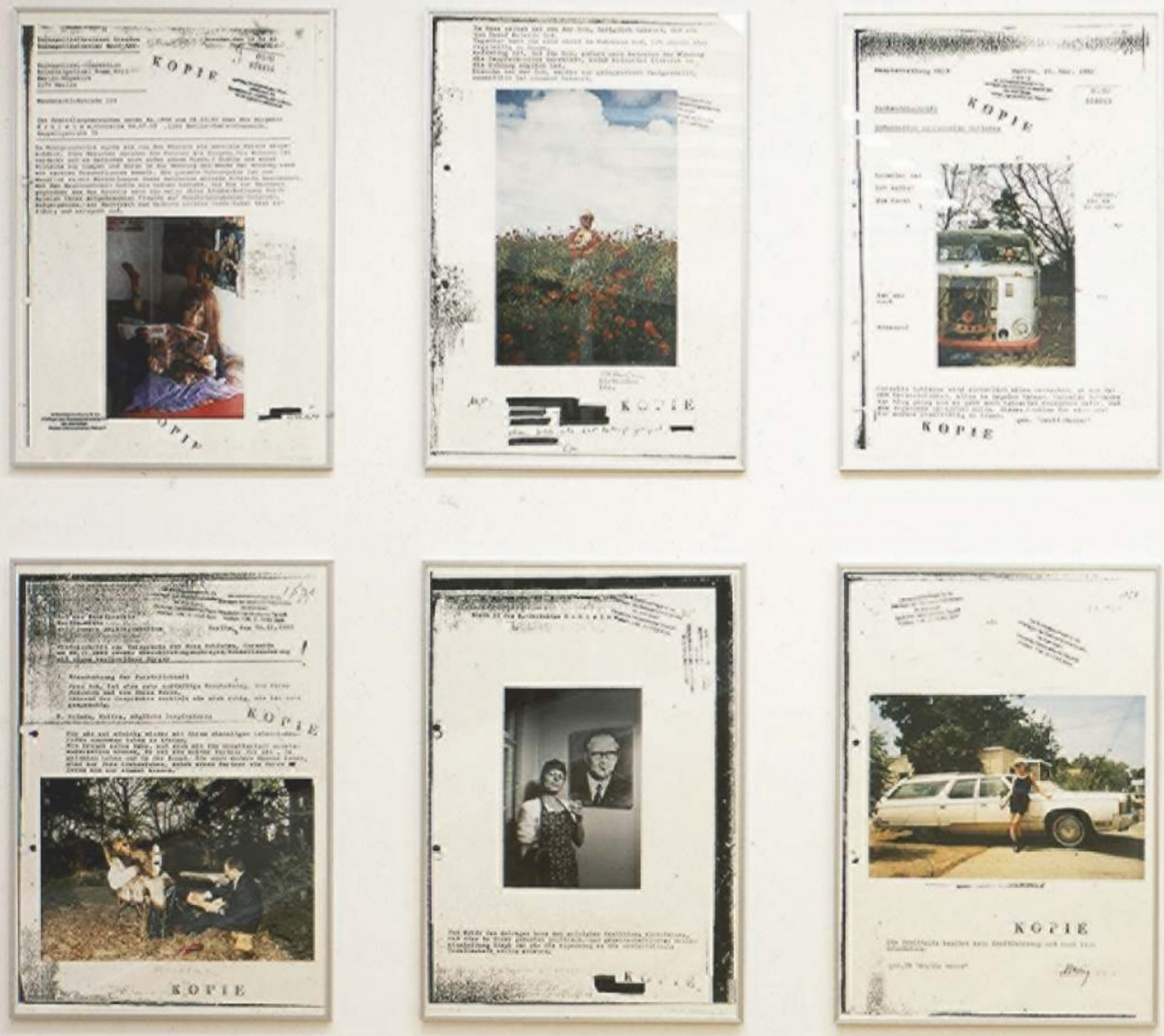

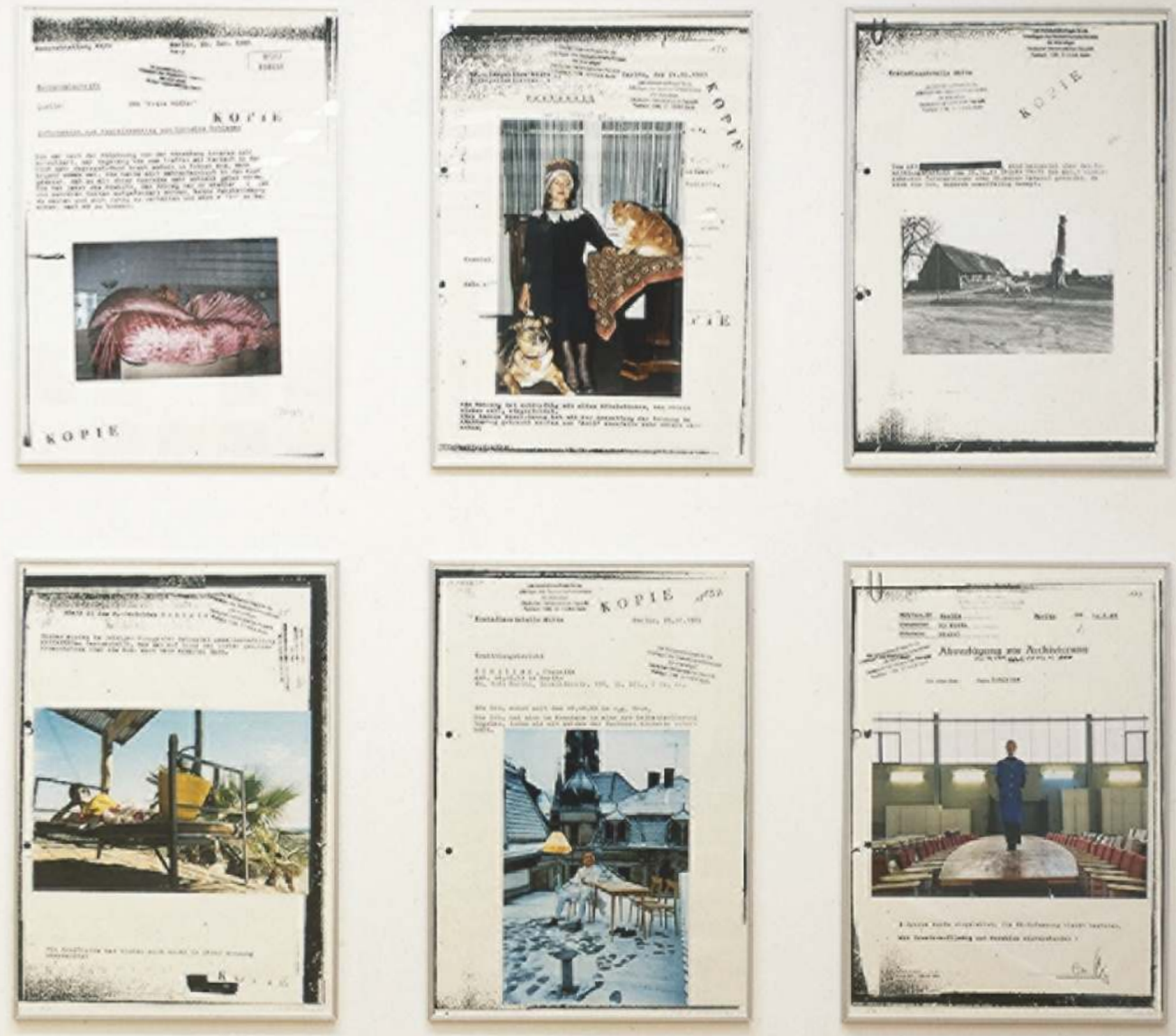
4. La version originale est extrêmement polysémique, ce qui rend sa traduction difficile. Nous en proposons ici une : 1) «Est artiste / moi-même / l'art / est-il / encore / présent ";

2) " est-ce qu'ils le / vivent / dans un / h- / ou / le font partir. » 3) «Est artiste / moi-même / vivent / l'art / est-ce qu'ils le / dans un / h- / est-il / ou / encore / le font partir / présent " ou encore «Est artiste / moi-même / vivre / l'art / le / dans un / h- / est-il / ou bien / encore / parti / présent ». [NdT]

\section{ci-contre}

fig. 5

Cornelia Schleime,

Bis auf weitere gute

Zusammenarbeit,

Nr. 7284/85, série

en 15 parties, 1993

photographie sérigraphiée,

$100 \times 70 \mathrm{~cm}$ (C) Cornelia

Schleime, avec l'aimable

autorisation de la galerie

Michael Schultz, Berlin. la BStU. Dans une encre plus foncée et tamponné de travers, le mot "KOPIE» en majuscules suggère un autre niveau d'intervention. Ces dossiers ont en effet été minutieusement examinés par les employés des archives, qui ont noirci certaines parties du texte où était révélée l'identité d'un tiers.

Cornelia Schleime intervient à différents degrés sur les textes. Les photographies qui les recouvrent constituent la part la plus incisive de son travail éditorial. Sur quelques planches, des zones de texte ont été déplacées ou coupées et remplacées par des espaces blancs. Dans un cas, l'autoportrait divise le contenu déjà fragmenté en deux (fig. 5). À gauche on peut lire: "Künstler ist / Ich selbst / die Kunst / ist sie / noch / anwesend 》 (1). À droite apparaissent les mots: "leben, / sie es / in einem / h- / oder / weg» (2). Réunies, les deux colonnes forment un poème visuel: "Künstler ist / Ich selbst / leben / die Kunst / sie es / in einem / h- / ist sie / oder / noch / weg / anwesend " (3) 4 . L'image coupe le texte en deux. Son contenu met littéralement en scène cette destruction: l'artiste apparaît au volant d'un camion. Quelques arbres créent un arrière-plan onirique. L'immense véhicule est coincé, immobilisé sur le chemin de terre qu'il essaie de franchir. Avec le capot ouvert et les fils qui pendent du phare avant gauche, il pourrait bien s'agir là d'une épave sortie d'une décharge.

Une remarque de l'informateur David Menzer est reproduite entièrement sous l'image, comme si le camion allait lui rouler dessus: "Cornelia Schleime fera tout ce qu'elle peut, légalement, pour sortir de la RDA. Cornelia Schleime est intelligente et rien n'indique qu'elle trouvera une issue, pour ellemême ou pour quelqu'un d'autre, en usant de la violence. " Cette observation, rédigée quelques mois après qu'on lui avait refusé sa première demande d'émigration, marque le début de plusieurs années de frustration. Cornelia Schleime quitta le pays en septembre 1984 après avoir effectué cinq demandes. L'autorisation lui fut accordée le lendemain du jour où, au téléphone avec un ami, elle avait menacé de commencer une grève de la faim publique (Schleime 2011). Cela faisait cinq ans que la Stasi la surveillait, mais l'État ne voulut pas courir le risque de voir sa frustration s'exprimer au grand jour. Le portrait met en image l'impossibilité de l'artiste de faire avancer sa cause par des moyens légaux. Dans un pays qui se définissait comme l'«État des travailleurs et des paysans ", l'image de Cornelia Schleime rentrant dans le moule en tant que conducteur de camion - et en tant que femme - renvoie bien au discours de l'État. Mais le véhicule, coincé dans un décor bien loin de l'univers industriel, est une allégorie de la frustration éprouvée par une artiste incapable d'avancer, même en suivant la voie bureaucratique.

Dans son analyse des publications populaires circulant en RDA, Irene Dölling a constaté, en s'appuyant sur les photographies représentant la femme au travail, que la domination masculine ne cessait pourtant d'exister et qu'on considérait en outre que c'était là une image déformée du sexe «faible»: "Bien que les photographies montrent que le fait d'avoir un emploi faisait partie de l'expérience quotidienne des femmes, elles donnent par ailleurs l'impression que le travail ne profite pas vraiment à leur "féminité". " (Dölling 1990: 40) À la fin des années 1980, la grande majorité des femmes occupaient un emploi ou suivaient une formation. Cependant, le travail était toujours considéré comme secondaire par rapport à la fonction maternelle. 


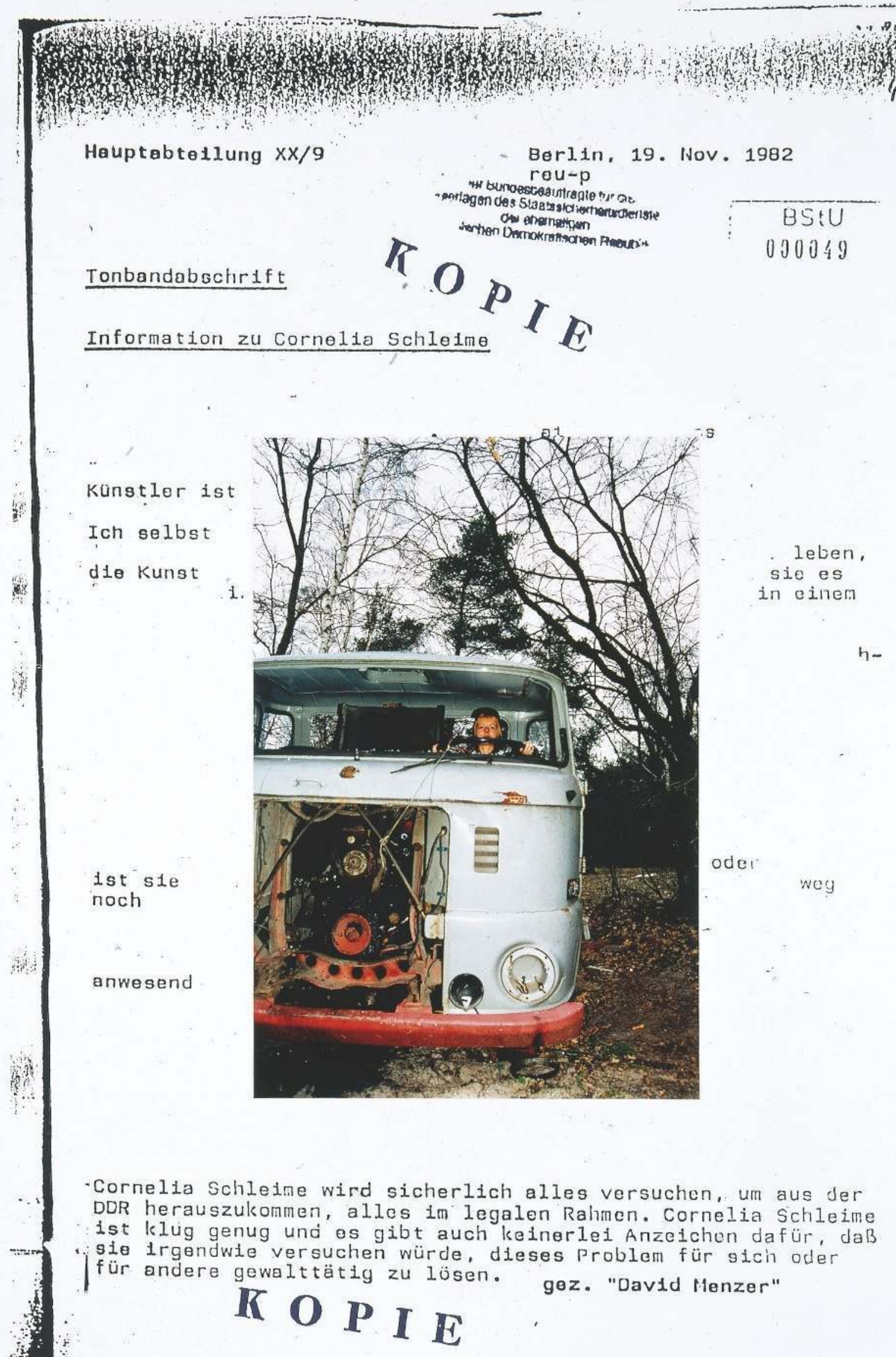


5. La littérature sur ce sujet est abondante. Voir Bathrick 1995 ; Boyer 2001. On mentionnera également deux films: von Krusenstjerna et Cederberg 1994 ; Hendel 2015.

6. Voici quelques dates sur le travail de Cornelia Schleime après qu'elle apprit le statut d'informateur d'Anderson. 1991: il est révélé publiquement qu'Anderson travaillait pour la Stasi depuis 1975 1993: création de la Série Stasi; 1994 : participation au documentaire Der Verrat (von Krusenstjerna et Cederberg) centré sur la figure d'Anderson:

2002 : publication de l'autobiographie d'Anderson; 2008 : publication du roman de Cornelia Schleime, Weit Fort; 2010 : l'artiste fait paraître In der Liebe und in der Kunst weiß ich genau was ich nicht will, une compilation de ses œuvres qui inclut des images et des notes sur la Série Stasi.

7. Anderson signe alors son rapport du nom de Fritz Müller, l'un de ses trois pseudonymes.

8. Bien qu'elle n'y révèle toujours pas l'identité d'Anderson, Cornelia Schleime est plus explicite sur la consternation qu'elle a éprouvée à son égard dans son roman semi-biographique, Weit Fort (2008).
En plus de signifier que les femmes étaient moins capables que les hommes, les photographies semblaient souligner que la beauté d'une femme dépend de la complexité de la tâche à effectuer (Dölling 1990: 43). Il est vrai en effet que peu de femmes occupaient des positions de pouvoir dans leur profession et que leur charge de travail était plus importante dans la sphère domestique. Ainsi que le suggère l'étude d'Irene Dölling, la culture photographique estallemande allait dans le sens de ce déséquilibre. On peut donc lire le message photographique de Cornelia Schleime comme un contrepoint à l'hypocrisie d'un pays fondé sur une idéologie égalitaire et qui, néanmoins, refusait que les femmes prennent les commandes.

La planche évoquée plus haut renseigne également - bien que de façon moins visible et plus autobiographique - sur la sournoiserie de l'État quand sa souveraineté était en jeu. David Menzer était l'un des noms de code utilisés par le poète et musicien Sascha Anderson, l'un des plus célèbres informateurs de la Stasi ${ }^{5}$. Pour Cornelia Schleime, la trahison d'Anderson fut particulièrement perverse: c'était un ami proche et un collaborateur artistique $^{6}$. Cette image, ainsi qu'une autre planche au moins de la Série Stasi faisant mention d'un rapport rédigé par Anderson 7 , atteste de cette trahison. Cependant, Cornelia Schleime décide de ne pas révéler son identité. C'est là un choix intéressant dans un travail qui consiste beaucoup par ailleurs à faire des révélations ${ }^{8}$. La décision de Cornelia Schleime est tout aussi anarchivistique que sa sélection de textes dévoilant indirectement l'identité d'Anderson et l'ampleur de sa tromperie. Cela étant dit, le rôle de l'artiste n'est pas de trahir, même ceux qui l'ont trahie. II ne s'agit pas là d'un projet visant à condamner des individus, mais plutôt une duplicité systémique. La Stasi était un problème collectif - ou plus exactement, un problème avec le collectif.

L'artiste pose devant une grosse Lincoln blanche garée dans l'allée qui mène à une maison de banlieue résidentielle (fig. 4). II fait beau. Dans le dossier on peut lire: "L'enquêtée ne possède pas de voiture ni de terrain. Signé IM “Martha Heine”. " Sur le portrait, Cornelia Schleime fixe l'objectif, le bras gauche appuyé sur le capot de la voiture. Elle porte des bottines noires, une minijupe serrée noire, un haut sans manches et une large ceinture violette. Elle a sur la tête une casquette blanche de capitaine, la jambe gauche repliée, le pied gauche caché derrière le droit. La pose suscite le désir, telle une séduisante publicité pour cette voiture et ce style de vie. Sur cette image, Cornelia Schleime met en scène des objets de consommation alors inaccessibles à la plupart des citoyens de RDA, en particulier à l'époque du rapport établi par la Stasi. Au milieu des années 1980, alors que ceux qui avaient les moyens de s'acheter une voiture devaient attendre parfois dix ans ou plus, l'État avait promis à tous les foyers est-allemands un véhicule pour 2010 (Bethke 1985: 61-62). Cornelia Schleime tourne en ridicule le consumérisme à la communiste de la RDA, fondé sur un épanouissement plus collectif qu'individuel. À l'inverse, elle se présente comme une capitaliste satisfaite posant avec sa voiture préférée. 


\section{L'imitation comme désobéissance}

Cette citation n'est pas un asservissement ou une simple réitération de l'original, mais une insubordination qui prend place au sein même des termes de l'original.

(Butler 2009: 58)

L'art anarchivistique respecte une logique formelle qui est pour l'essentiel celle de l'archive, car, comme l'observe Foster, il «ordonne souvent les matériaux selon une logique quasi archivistique » et, par cette pratique citationnelle, "souligne le fait que la nature des matériaux d'archive est d'être trouvés et néanmoins construits, factuels mais fictifs, publics et privés" (Foster 2004: 5). Chez Cornelia Schleime, la qualité fictive ou construite de l'archive est mise en lumière par la mimésis. Le titre formel de l'œuvre, Dans l'attente de la poursuite d'une bonne collaboration, Nr. 7284/85, singe en effet le langage de la police secrète. La remarque introductive de l'exposition s'ouvre quant à elle sur les remerciements d'usage: "Ce travail n'aurait pas pu voir le jour sans l'aide du ministère de la Sécurité d'État de la RDA ainsi que ses nombreux informateurs, qui ont rédigé avec beaucoup de minutie ces textes. Je leur exprime ici toute ma gratitude.»

Cornelia Schleime ne conteste pas la parole de ses oppresseurs. Elle l'utilise, la cite et va même jusqu'à l'incarner dans ses autoportraits. Dans Ces corps qui comptent, Judith Butler théorise la résistance potentielle implicite dans ce type de citation consciente et critique du pouvoir (Butler 2009). En analysant dans le détail la manière dont Cornelia Schleime manifeste les désirs de l'État est-allemand tels qu'ils sont projetés sur l'image fantasmée de la femme communiste libérée, on retrouverait certainement l'usage spécifique que Judith Butler fait de sa théorie pour décrire la performance du genre. Cependant, la Série Stasi évoque plus largement le langage de l'appareil de surveillance de la RDA, c'est-à-dire sa forme et sa texture, ainsi que sa façon de se servir de l'observation concrète et du jugement. La personnification de la résistance chez Judith Butler rappelle l'impulsion performative de Cornelia Schleime:

[D]'accord, très bien, de toute façon, je ne voulais pas faire partie de votre économie, et je vais vous montrer ce que ce réceptacle inintelligible peut faire à votre système. Je ne veux pas être une copie dégradée de votre système, mais je vous ressemblerai quand même en mimant les textes par lesquels vous construisez votre système et en montrant que ce qui ne peut y entrer est en fait déjà à l'intérieur de lui (comme son extérieur nécessaire). (Ibid. : 58)

Dans la Série Stasi, Cornelia Schleime reste ce réceptacle inintelligible. Cette ontologie lui est attribuée par l'État - son extérieur nécessaire -, qui ne peut lui faire une place dans son système binaire (capitaliste/communiste; révolutionnaire/fasciste). En se transformant en réceptacle, elle incarne les identifications multiples qui frustrent cet État. L'ironie est évidente: Cornelia Schleime devient ingérable en devenant la femme que la Stasi voulait qu'elle soit. La revendication biopolitique et idéologique du pouvoir de la RDA, à savoir sa classification des individus par catégories et par comportements afin de créer le citoyen idéal, est ainsi mise en avant et ridiculisée dans son 


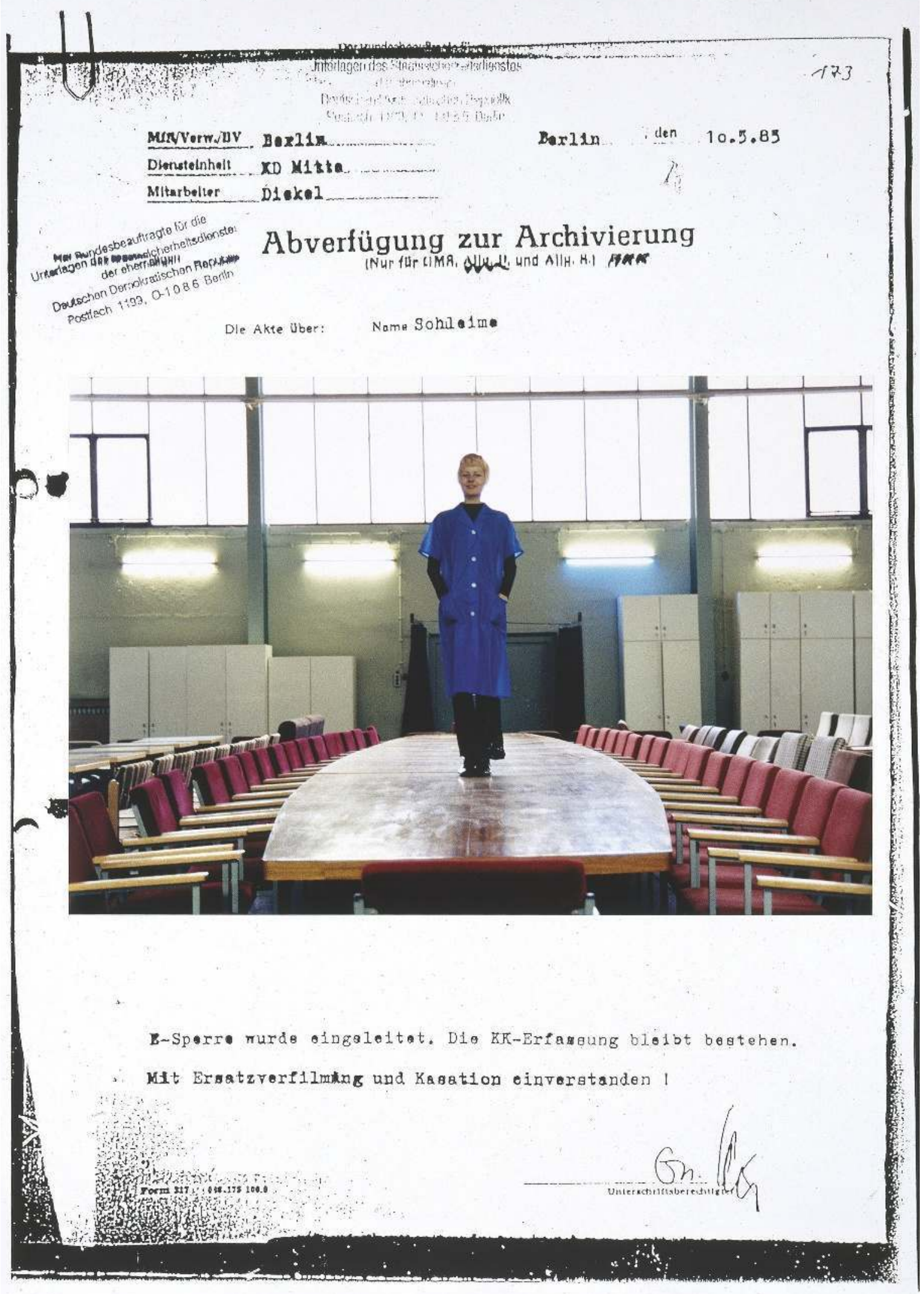


incohérence fondamentale. Si Cornelia Schleime réutilise son dossier de la Stasi de manière «anarchivistique», pour reprendre la logique de Foster, c'est également là un geste qui met au jour le chaos comme condition préexistante à l'archive. Les documents d'archive produits pendant des années d'oppression deviennent dès lors la preuve de l'échec inévitable de l'État. C'est au moment où Cornelia Schleime est ce que la Stasi voit en elle qu'elle révèle l'absurdité de l'État.

L'idée développée par Judith Butler de «citation performative » décrit des actions quotidiennes, l'énoncé (utterance) pouvant également servir à théoriser des œuvres d'art proposant une mise en scène des langages de l'oppression. L'artiste Guillermo Gómez-Peña, par exemple, met en actes les stéréotypes de race et de genre projetés sur les corps latinos afin de souligner leur prévalence dans la culture normative. En plus de ses performances en direct, l'artiste crée des «photo-performances», un terme qu'il a imaginé pour décrire la relation entre les tableaux vivants pour lesquels il pose et la logique visuelle de sa pratique artistique. Les photo-performances mettent l'accent sur l'objectification de la figure humaine devant l'appareil. Comme l'explique Jennifer González, «l'accent est mis sur l'interface entre les performers et l'appareil, sur l'acte de poser en lui-même, sur le dialogue à la fois intime et public qui s'instaure dans la relation entre la lentille et le corps» (González et Gómez-Peña 2009: 238). Les poses excentriques de Gómez-Peña recréent des scènes tout aussi familières que déconcertantes pour le spectateur. II joue à l'Indien, se couvre de plumes et de peintures de guerre et reste stoïque face à l'objectif. À cette mise en scène de l'exotique, il ajoute d'autres stéréotypes du corps mexicains, qu'on trouve rarement mêlés aux premiers: il porte ainsi un fusil d'assaut et devient narcotrafiquant. Des aliens et des cyborgs font leur apparition, références évidentes aux «indésirables» de sa race. Ce sont là, dit-il, des «personnages de performance complexes. Le corps [est] paré, travaillé, truffé de références culturelles et politiques » (ibid. : 241).

La politique de la représentation est évidemment ici différente de celle de la Série Stasi. Si Cornelia Schleime pose par ailleurs presque toujours seule, Gómez-Peña fait intervenir d'autres personnages pour évoquer une condition culturelle partagée, déclenchant ce que González appelle «des glissements d'identité » (ibid. : 238). Dans la Série Stasi, ces glissements se manifestent à travers les différentes identités projetées par l'État sur le corps de l'artiste: c'est une mère-travailleuse-enfant-sexy-poufiasse. Ses personnages sont animés par la présence de ceux qui l'observent - ceux dont la présence est dissimulée dans le texte et ceux qui regardent la série aujourd'hui. L'équation proposée par Gómez-Peña pour décrire ses photo-performances - «un quart de stéréotypes, un quart de projection de la part des spectateurs, un quart de réalité sociale et un quart d'artefact esthétique " (ibid.: 249) fait écho aux multiples discours qui sont la cible de Cornelia Schleime. La Série Stasi, en insérant des autoportraits dans des archives de surveillance, exprime de manière littérale le sentiment d'anxiété qui résulte du fait d'être regardé, classé et évalué.

Ni Schleime ni Gómez-Peña ne succombent évidemment à leurs observateurs. Le quart esthétique de l'équation citée plus haut renvoie à

\footnotetext{
ci-contre

fig. 6

Cornelia Schleime,

Bis auf weitere gute

Zusammenarbeit,

Nr. 7284/85, série

en 15 parties, 1993, photographie sérigraphiée, $100 \times 70 \mathrm{~cm}$ (C) Cornelia Schleime, avec l'aimable autorisation de la galerie Michael Schultz, Berlin.
} 
9. Voir le travail de Mark Fenemore sur la culture du rock'n'roll dans les années 1950 et 1960 en RDA, en particulier son chapitre "Manufacturing Consent", qui étudie la façon dont l'État s'est approprié des formes occidentales modernes de culture afin de domestiquer - et de contrôler - une jeunesse privée de ses droits (Fenemore 2007 : 156-183). l'agentivité de l'artiste qui fabrique un objet autobiographique - ce qu'il ne faut pas oublier quand le contenu des sources utilisées pourrait avoir tendance à éclipser la présence de choix artistiques. L'humour et l'ironie jouent à cette fin un rôle central chez les deux artistes. Pour Cornelia Schleime, l'humour est un mécanisme de défense et de protection au moment d'exposer publiquement l'oppression dont elle est l'objet (Schleime 2010: 75). De même, Gómez-Peña s'en sert pour révéler des réalités sociales tragiques et hostiles. II s'agit là, comme le décrirait Judith Butler, de «déplacer le déplacement ": l'imitation d'un imaginaire oppressif, qu'il concerne la race, le genre ou l'idéologie, expose le fait que cette ontologie est «un "effet" d'une ruse du pouvoir» (Butler 2009: 59).

Cornelia Schleime amplifie parfois de manière exagérée les jugements portés sur son mode de vie décadent, qui deviennent ainsi le signe de la vulnérabilité de l'État, et non celui d'un échec personnel. Sur une image associée à une observation datant de 1982, Cornelia Schleime porte une longue perruque rousse et lit Bravo, un magazine pour adolescents populaire en Allemagne de l'Ouest (fig. 7). Allongée sur le ventre, elle balance les jambes avec désinvolture. La couleur de sa tenue reprend celle du magazine, dont la quatrième de couverture reproduit un portrait de Christian Slater, idole américaine des années 1990. À sa gauche, le mur est couvert d'affiches de Cinderella, un groupe américain de glam métal dont le premier album date de 1986. Le décalage temporel entre le rapport de 1982 et ces artefacts de la culture pop occidentale illustre les inquiétudes du gouvernement est-allemand quant à l'influence exercée par l'Ouest, mêlées à une grande angoisse par rapport à l'avenir ${ }^{9}$. Cornelia Schleime est ici l'image de la décadence occidentale, un signe des temps à venir.

Ce rapport de 1982 est l'un des plus longs de la Série Stasi. C'est aussi l'un des seuls à faire référence au travail de Cornelia Schleime en tant qu'artiste et musicienne:

Les locataires du rez-de-chaussée la prennent pour une asociale. Ses visiteurs entrent par la fenêtre. L'appartement est entièrement calfeutré, et à l'exception d'une table, de deux chaises et d'un lit de camp, tout n'est que tas de chiffons et désordre. Sur les murs de l'appartement sont peintes des figures féminines nues. Aux dires de ses connaissances, la porte de la pièce principale est entièrement bariolée, de haut en bas, avec des pastels. Elle n'a aucun contact avec ses voisins. Elle sort seulement de sa coquille quand, à cause du bruit qu'elle fait la nuit avec ses amis en jouant de la musique (de la guitare, de la basse, etc.), on la rappelle à l'ordre pour tapage nocturne. Quand cela se produit, elle se montre grossière et arrogante.

La photographie dépasse toutes les espérances de l'informateur. Elle traduit ces observations en se représentant comme un cliché tout droit sorti de son magazine, et devient ainsi un tableau vivant de la culture adolescente occidentale. Les rapports de la Stasi sont hyperboliques, ainsi que l'écrit Schleime dans son roman semi-autobiographique Weit Fort: "Ce n'est pas un lit de camp, [...] mais un lit simple tout à fait normal. Et le désordre évoqué n'est rien d'autre qu'une pile de toiles. Mais la femme écrit ces lignes de 
son point de vue à elle. [...] Elle veut peut-être tout simplement faire plaisir à ses supérieurs [...] et invente une histoire tirée par les cheveux. 》 (Schleime 2008: 34) On peut aussi imaginer que l'officier de la Stasi qui reçut et résuma le rapport de la voisine ait pris certaines libertés afin que Schleime corresponde à un certain type. Dans son étude sur la police secrète de la RDA, Jens Gieseke explique que les rapports étaient souvent modifiés pour répondre aux opinions et aux besoins des leaders du parti (Gieseke 2014: 112). La Stasi était particulièrement attentive aux représentants de cultures marginales, sur lesquels l'État avait peu de visibilité (ibid. : 113).

Dans son autoportrait, Cornelia Schleime épouse les jugements de son observateur. Elle développe notamment toutes les représentations associées au terme «asocial» : insulte par excellence en RDA, le mot pouvait avoir des répercussions judiciaires bien réelles. Être asocial ne signifiait en effet pas seulement être distant ou s'habiller et vivre en dehors de la norme, mais pouvait aussi renvoyer au fait d'être au chômage, ce qui était illégal et répréhensible. Selon le paragraphe 249 du Code pénal de la RDA, connu sous le nom de Assi-Paragraphen, les citoyens valides sans emploi pouvaient être condamnés jusqu'à un maximum de deux ans d'emprisonnement. Même si, comme le précise Paul Kaiser, l'application de cette loi ne fut pas systématique et combinée en réalité à d'autres infractions, «elle eut des effets dissuasifs durables » (Kaiser 2009: 110). Les citoyens anticonformistes étaient exposés aux manipulations, aux mensonges et à l'animosité de leurs observateurs. Cornelia Schleime n'avait pas d'emploi stable ou traditionnel, mais le syndicat des artistes professionnels la protégeait, jusqu'à ce qu'elle rende sa carte en 1982. Comme bien d'autres artistes d'avant-garde de la RDA, Schleime faisait peu de cas de la législation et des mœurs sociales au cœur du fantasme idéologique de l'État. Sur la photographie décrite plus haut, elle se délecte de ne faire aucun compromis. Dans une tenue excessivement colorée, absorbée dans sa lecture de niaiseries, Schleime devient la femme que la Stasi voulait et avait même besoin qu'elle soit: la citoyenne tombée dans la décadence occidentale, que la RDA devait sauver. C'était l'incarnation de l'ennemi, la preuve dont la Stasi avait besoin pour justifier sa propre existence.

Dans la série, une seconde planche, tout aussi réprobatrice, met en évidence la manière dont le fantasme et le désir alimentaient les opérations de surveillance de la Stasi. Une image en noir et blanc divise en deux le texte d'un document non daté extrait du dossier de l'artiste (fig. 8). Cornelia Schleime est étendue en travers d'un lit double. Elle est habillée, mais a écarté les jambes et découvert ses cuisses. Entre les deux doigts de sa main gauche, elle tient une cigarette allumée. Sur le lit se trouvent également un cendrier rond en verre et un téléphone à touches. Elle compose un numéro avec le gros orteil de son pied gauche et tient le combiné avec son épaule. La pose est séductrice et langoureuse. Cornelia Schleime ne regarde pas l'objectif. Sous ce cliché figurent ces lignes: «La Sch. gagne apparemment beaucoup d'argent avec son métier. L'enquêtée est bien habillée. Elle porte aussi des "vêtements occidentaux". Personne dans l'immeuble n'a encore jamais vu de petit ami. " L'artiste met ici en scène le fantasme de la femme célibataire bien habillée qui a été séduite par les artifices de l'Ouest. Le téléphone, objet très rare en RDA, est l'ultime signe de luxe et de loisir. Sa robe moulante est tout l'inverse de la tenue fonctionnelle page suivante

fig. 7

Cornelia Schleime, Bis auf weitere gute Zusammenarbeit, Nr. 7284/85, série en 15 parties, 1993, photographie sérigraphiée, $100 \times 70 \mathrm{~cm}(C)$ Cornelia Schleime, avec l'aimable autorisation de la galerie Michael Schultz, Berlin. 


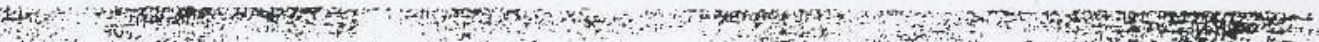

Volkspolizeikreisamt Dresden Volkspolizeirevier Nord-ABV-

Volkspolizei-Inspektion

Kriminalpolizei Romm.VIII

Berlin-Köpenick

1170 Berlin

Wendenschloßstraße 130



Ihr Ermittlungsersuchen unter Az.:958 vom 09.03 .82 über die Bürgerin s c h 1 e $i \mathrm{~m}$ e, Cornelia 04.07 .53 , 1160 Berlin-Oberschöneweide, Zeppelinstraße 79

Im Wohngrundstück wurde sie von den Mietern als asoziale Person eingeschätzt. Ihre Besucher nutzten die Fenster als Eingang.Die Wohnung ist verdeckt und es befindet sich auBer einem Tisch,2 Stühle und einer Pritsche nur Lumpen und Unrat in der Wohnung.Die Wände der Wohnung sind mit nackten Frauenfiguren bemalt. Die gesamte Wohnungstür ist zum Hausflur zu,mit Mitteilungen ihrer Bekannten mittels ôlkreide beschmiert Mit den Hausbewohnern hatte sie keinen Kontakt. Sie kam nur Nachbarn gegenüber aus der Reserve wenn sie wesen ihrer Lärmbelästigung durch Spielen ihrer mitgebrachten Freunde auf Musikinstrumenten-Gitarren, Baßgeigenusw.-zur Nachtzeit zur Ordnung geruien rurde.Dabei trat sio fläzig und arrogant auf.

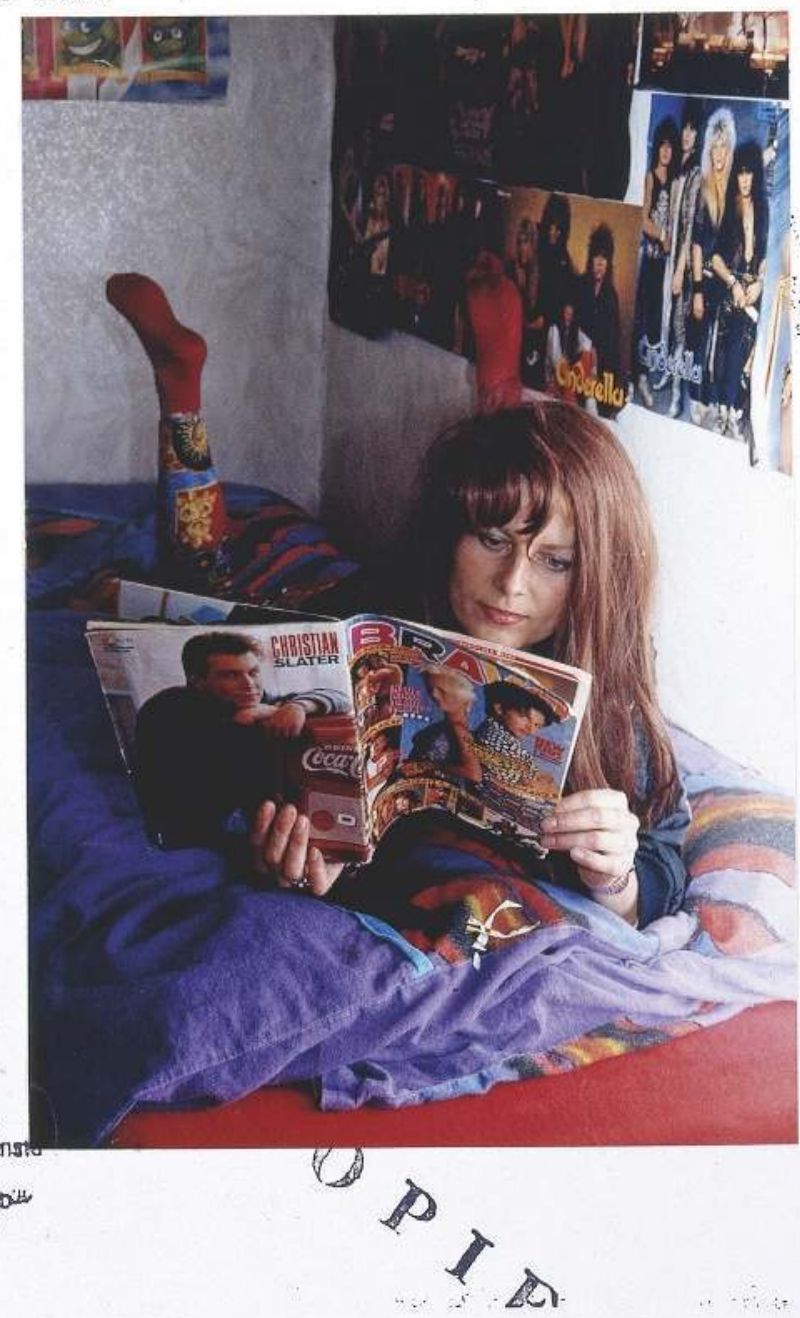

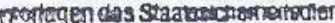
or mentrom

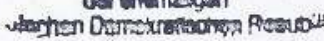




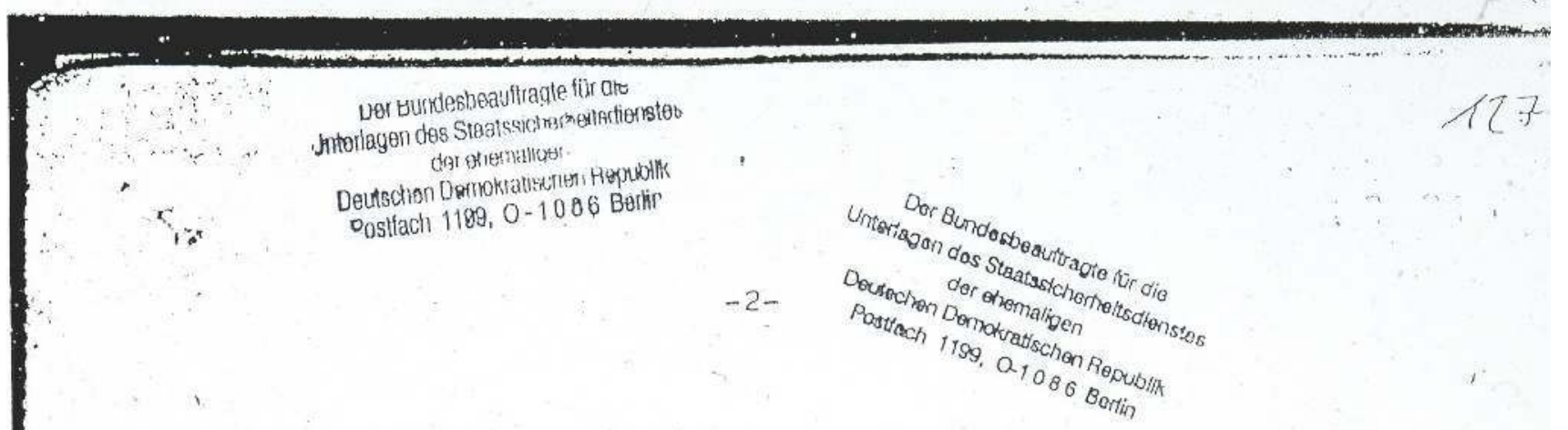

In Wohnhaus ist die zu Ermittelnde den Mietern nicht bekennt, nur der iKP.

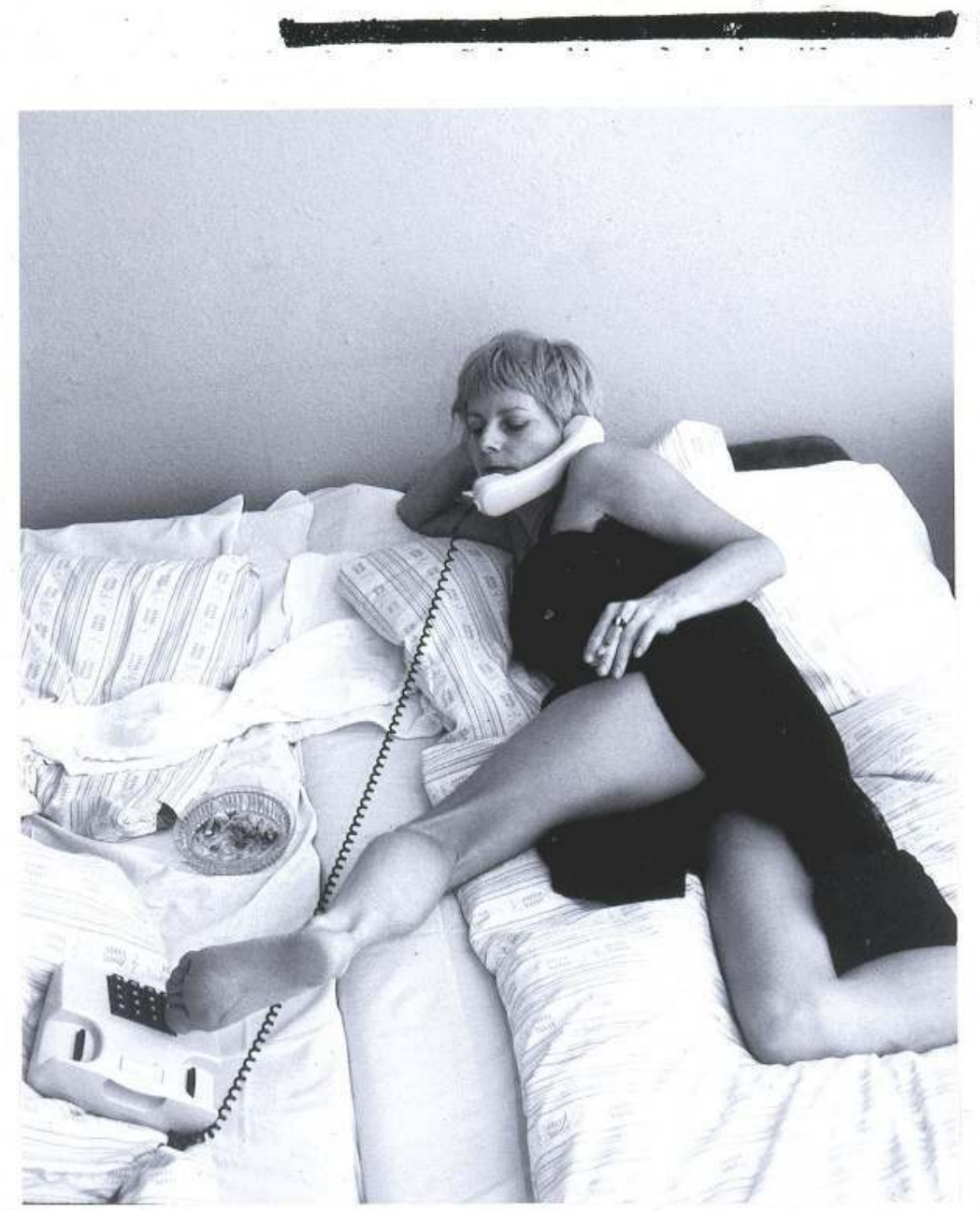

Die Sch. soll in ihren Beuf auch ganz gut verdienen.

Die Ermittelte ist gut gekleidet, sie trägt auch "westkleidung". II $O$ 直

im Haug noch nicht gegehen. 
page

précédente

fig. 8

Cornelia Schleime,

Bis auf weitere gute

Zusammenarbeit,

Nr. 7284/85, série

en 15 parties, 1993

photographie sérigraphiée,

$100 \times 70 \mathrm{~cm}$ (C) Cornelia

Schleime, avec l'aimable

autorisation de la galerie

Michael Schultz, Berlin. du citoyen modèle. Dans la marge gauche du rapport, une marque signale la phrase «Personne dans l'immeuble n'a encore jamais vu de petit ami». Ceci révèle une stratégie de surveillance spécifique, à savoir la pratique de la Zersetzung (décomposition), qui visait les relations personnelles des individus surveillés (Gieseke 2014: 147). On aurait pu recruter le petit ami comme informateur, le persuader de faire des rapports sur Schleime ou bien l'observer lui-même afin d'obtenir davantage d'informations. L'usage que Schleime fait du texte souligne clairement la scopophilie de la Stasi. L'intérêt de l'État pour la vie sexuelle de l'artiste est représenté comme une forme de voyeurisme légitime.

Cette image en particulier rappelle le projet Untitled Film Stills de Cindy Sherman (1977-1980), qui proposait lui aussi une parodie du voyeurisme. Chaque Film Still est une scène qui met en jeu la nostalgie de la culture cinématographique américaine des années 1950. À travers ces images, Cindy Sherman propose la performance des archives de la culture pop de l'époque. L'artiste revêt différentes tenues pour répondre au regard désirant du spectateur. Les Film Stills, qui sont une critique subtile de l'immuabilité absolue caractérisant l'image idéalisée de la femme moderne américaine, se jouent des spectateurs en leur faisant voir leur propre désir de prendre les femmes pour des objets. Comme le remarque Laura Mulvey, «en insistant exagérément sur la surface, le projet laisse peu à peu entrevoir que celle-ci pourrait masquer quelque chose devant rester caché, et l'indice d'un autre espace se met alors à affleurer à l'intérieur d'une façade trop évidente" (Mulvey 1991: 141). Laura Mulvey replace la série dans le contexte explicite de la lutte féministe aux États-Unis: elle met en images "une culture particulière de l'apparence, spécialement de l'apparence féminine ", produite par la culture de masse (ibid.). Sherman cite ce langage visuel pour montrer comment celui-ci saisit à la fois l'objet du regard et le spectateur comme regardant. En outre, et pour revenir encore une fois à Judith Butler, Cindy Sherman met en lumière l'iniquité de ce langage normatif du regard et, de cette manière, parvient à le déplacer, à le déstabiliser et le discréditer.

Il est à bien des égards important de prendre en compte le contexte historique des Film Stills, mais ce n'est pas absolument indispensable. Cependant, une comparaison entre les travaux de Cindy Sherman et de Cornelia Schleime s'avère rapidement difficile dès lors qu'on considère les différences entre les moyens de production et les contextes nationaux qui les ont vus naître. Bien évidemment, la façon dont Schleime pose pour le spectateur suggère que les femmes est-allemandes étaient soumises à une objectification tout à fait semblable à celle des femmes américaines décrites par Cindy Sherman. Mais tandis que les photographies de Sherman forment un ensemble qui reproduit le langage de l'objectification des femmes et, en dernier lieu, démontrent que ce langage est au fondement de la culture pop américaine, celles de Schleime délivrent un message plus spéculatif. En 1993, quand Schleime présenta pour la première fois sa Série Stasi à l'exposition Art Cologne, les gens lui demandèrent où elle avait trouvé les textes. "Cette question, dit-elle, m'a fait comprendre que les artistes de l'Ouest travaillent très différemment, c'est-à-dire de manière virtuelle, alors que les artistes de l'Est travaillent à partir de leur propre expérience. ” Si la politique de la représentation constitue à n'en pas douter un sous-texte 
important, la Série Stasi véhicule des réalités matérielles, évoque des destinées bien réelles et donne à voir des effets non pas virtuels mais soigneusement documentés.

Les images de Cornelia Schleime sont également différentes parce qu'elles ne condamnent pas ceux qui les regardent aujourd'hui. Au contraire, le but de l'artiste est de proposer une histoire qui dénonce les informateurs de la Stasi et leur participation à un système de délation. Cette intention se lit implicitement dans le titre officiel de l'œuvre, Dans l'attente de la poursuite d'une bonne collaboration. Cette expression, qui reprend la rhétorique d'une bureaucratie inefficace et maladroite, souligne le fait que la Stasi dépendait des citoyens qui espionnaient leurs collègues, voisins, amis, amants et membres de leur famille. Les remerciements que Cornelia Schleime adresse en introduction au «ministère de la Sécurité d'État de la RDA et à ses nombreux collaborateurs " sont une autre marque du dessein de l'artiste, le sarcasme de la formule cachant mal son amertume.

«L'agent de liaison XXXX n'a pas reçu de nouvelle information depuis le rapport d'enquête du 24/10/83 (pages 74-75 du document) car la Sch. s'est montrée très discrète dans ses mouvements. » (fig. 9) Schleime se représente sur une route de campagne devant la maison d'un agent de la Stasi. Elle est habillée un peu comme une enfant, avec une jupe courte à fleurs, des collants foncés et une veste de flanelle rouge. Elle tire derrière elle une poussette qui est attachée à son immense tresse blonde. L'accoutrement choisi par l'artiste incarne de multiples registres de la féminité. Elle représente une petite fille qui est aussi une mère, faisant ainsi référence à la double tâche qui était celle des femmes en Allemagne de l'Est. II était certes devenu plus facile pour les femmes actives d'élever leurs enfants; mais l'État n'en attendait pas moins qu'elles travaillent, et cela sans redéfinir les normes parentales qui laissaient les femmes faire seules les frais de la charge supplémentaire qui les attendait à la maison (Ferree 2012: 48). Dans l'espace qui sépare le corps de Schleime et la poussette, l'artiste a glissé à l'arrière-plan l'image d'un pays bien présent mais quelque peu désuet. La RDA manifeste toute l'exigence de sa dépendance à l'égard de l'artiste, qu'elle lui impose. La photographie réunit deux temporalités: celle du présent et de l'avenir du pays. En 1983, à l'époque du rapport, Schleime est une artiste dont le pays interdit tout mouvement. En 1993, elle croule sous le poids du passé de ce pays, qui lui revient à travers les centaines de pages de son dossier. La pose de Schleime est rigide. Elle hésite entre ces deux temporalités. Elle a quitté les années 1980, mais elle y est encore prise au piège. En associant cette photographie à un texte où il est dit que ses actes ne sont pas dignes d'être consignés, Schleime demande aux spectateurs actuels de la voir telle qu'elle est aujourd'hui, de reconnaître qu'elle va de l'avant et d'accélérer pour elle le mouvement.

\section{Le temps de l'archive}

Dans la Série Stasi, les photographies de Schleime sont présentées comme des suppléments aux rapports de surveillance dont elle fut l'objet pendant des années. Les clichés sont combinés aux documents écrits afin 


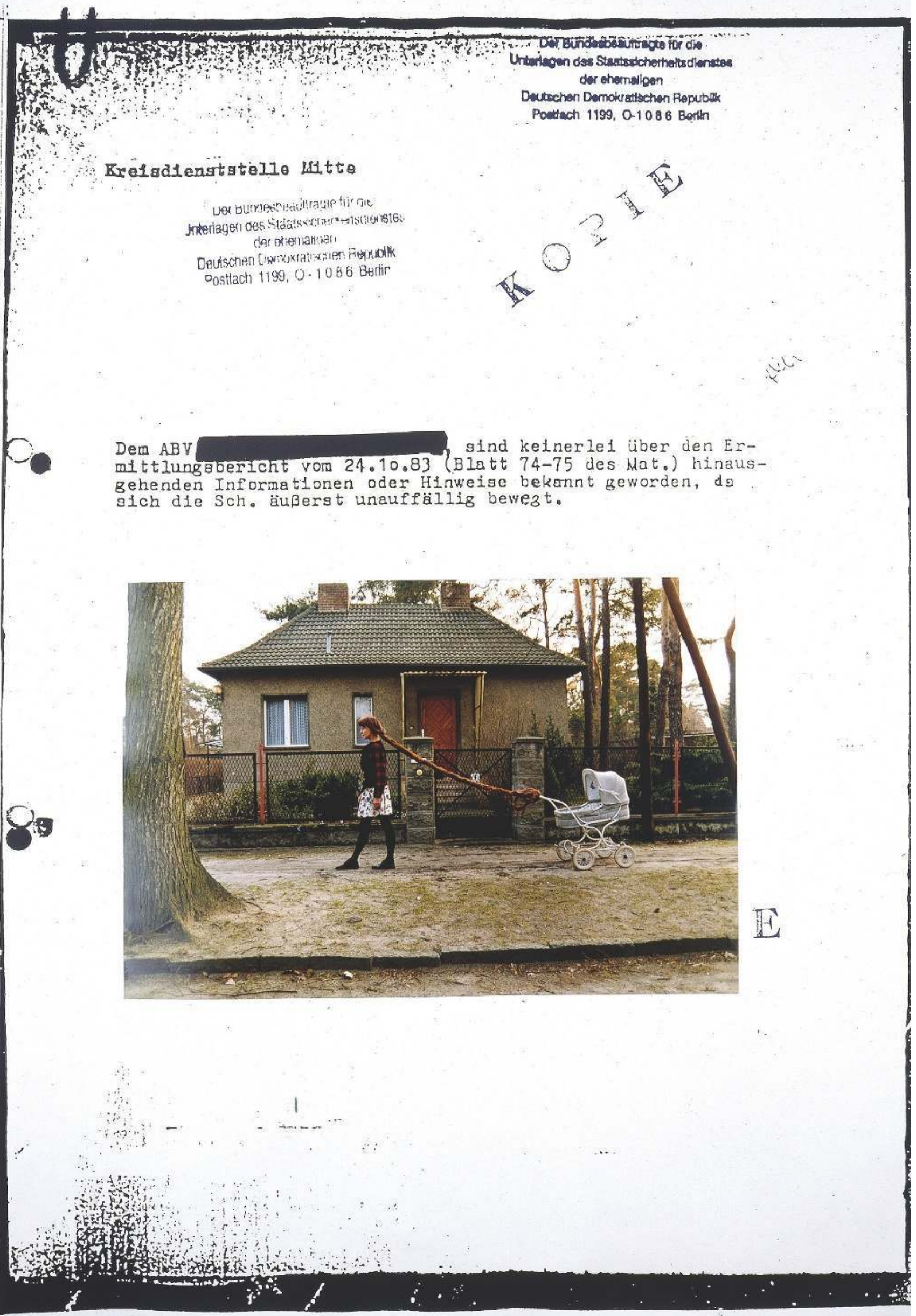


d'amplifier les archives. Et c'est dans la tension produite par ce geste que réside l'articulation conceptuelle la plus importante du projet. Chaque image interagit avec le texte du dossier pour révéler les réalités et les faiblesses du système étatique de surveillance et de gouvernance.

Nombreux sont les artistes qui, avant Schleime, ont permis de penser les différentes nuances de l'espionnage. II existe notamment une longue histoire de femmes artistes ayant utilisé les techniques de surveillance pour mettre en exergue la relation entre genre et observation. Pour le dire plus explicitement, et peut-être de manière trop simpliste, elles ont regardé comment les femmes étaient observées. La série de photographies de Sophie Calle, The Shadow, créée en 1981, ainsi que la performance intitulée Roberta Breitmore de l'artiste Lynn Hershman Leeson (1973-1978) offrent de nombreux points de convergence. À l'image de la Série Stasi de Cornelia Schleime, ces œuvres interrogent ce que cela signifie d'être observé en développant une thématique parallèle, celle des personnalités multiples.

Pour The Shadow, Sophie Calle avait demandé à sa mère d'engager un détective privé pour la suivre. Le projet est composé de deux séries de documents d'archives produisant «deux visions parallèles du même jour ", comme l'explique Sophie Calle (Calle 1993: 33). Dans la première, l'artiste a consigné ses mouvements et ses actions, ainsi qu'un autoportrait. La seconde contient les rapports du détective et une série de clichés de l'artiste. Pour Sophie Calle, ce projet servait de contrepartie à un travail antérieur dans lequel elle avait suivi des étrangers et noté leurs mouvements: «Dans ce projet, je m'étais tant impliquée dans cet acte de suivre les autres que j'ai voulu, d'une certaine manière, inverser la relation. » (Ibid. : 32) The Shadow n'est pas né de la même idée que l'œuvre de Schleime, mais il en adopte la forme. Dans les deux cas, les artistes observent l'observateur et l'expérience d'être observé. Cependant, il existe des différences frappantes en matière de pouvoir et d'agentivité. Là où Sophie Calle réalise son travail dans le cadre des droits dont elle jouit en tant que citoyenne française, notamment la liberté de mouvement ou le droit d'être volontairement placée sous surveillance, Cornelia Schleime ne peut quant à elle échapper à sa condition de citoyenne sous surveillance.

Pour ce qui est de la temporalité, le parallèle est plus clair avec la performance de Lynn Hershman Leeson. Roberta Breitmore est le personnage, l'alter ego que s'est créé Leeson. L'intérêt de ce projet réside dans l'utilisation des infrastructures publiques afin de rendre plus réel cet alter ego. Pour incarner Breitmore, Leeson s'est transformée physiquement: elle s'est habillée différemment, a porté des perruques et a modifié sa personnalité. Elle a réussi à obtenir un permis de conduire, des radiographies dentaires et un compte en banque, elle a fait paraître des petites annonces pour faire des rencontres. Des photographies de surveillance ont même été prises lors de ses différents rendez-vous. Elle a également pris des notes sur son expérience émotionnelle en tant que Breitmore. Tous ces documents prennent la forme de journaux intimes et de lettres rédigées par le psychiatre de Breitmore. Amelia Jones parle pour ce projet de «mascarade» (masquerade) et prend l'exemple de la plus célèbre parmi les premières apparitions publiques de Breitmore pour illustrer le rôle important de la surface dans la politique de l'œuvre:

\section{ci-contre}

fig. 9

Cornelia Schleime,

Bis auf weitere gute

Zusammenarbeit,

Nr. 7284/85, série

en 15 parties, 1993,

photographie sérigraphiée,

$100 \times 70 \mathrm{~cm}$ @ Cornelia

Schleime, avec l'aimable

autorisation de la galerie

Michael Schultz, Berlin. 
«Dans Roberta Construction Chart \#2 [un portrait photographique sur lequel figurent des indications pour le maquillage de Lynn en Roberta], [Leeson] présente de manière littérale les moyens par lesquels elle devient son alter ego. En renseignant ainsi cette transformation subjective, elle montre avec ironie la facilité apparente d'une telle transformation et, dans le même temps, le caractère superficiel d'un tel changement "cosmétique". » (Jones 2005: 110) Tout comme The Shadow, la performance de Leeson insiste sur l'expérience qui consiste à devenir un sujet à travers les yeux d'un observateur. Selon Jones, «l'exposition de soi par le simulacre n'est pas un outil ou une stratégie que l'on peut adopter ou rejeter à volonté. Au contraire, en tant que composant de la mascarade féminine, c'est un élément central de l'expérience des femmes dans le patriarcat » (ibid.). De manière semblable, la Série Stasi explore la façon dont l'identité est définie par la surface du corps et par l'observation de cette surface. II y a pourtant une différence fondamentale entre les photographies de Schleime et la performance de Leeson. Chez la première, la construction du genre par la pratique de la surveillance n'est qu'un élément de l'appareil de pouvoir étatique dont elle est victime. Son travail vise moins à dénoncer les inégalités de genre qu'à révéler les conflits et le désordre au cœur du système.

D'un point de vue historique, il est peut-être plus pertinent (ou en tout cas plus en accord avec les motivations de l'artiste) de comparer la Série Stasi à d'autres œuvres qui explorent la surveillance comme condition matérielle. Les artistes contemporains travaillant sur ce thème sont légion: Hito Steyerl, Trevor Paglen, Walid Raad et Jenny Holzer, pour ne citer qu'eux. Comme Schleime, ils s'intéressent à la question de l'exposition au regard. Cependant, ils envisagent différemment le rôle de l'observateur dans l'intégration ou l'internalisation du matériau produit par l'observation. Prenons l'exemple de Holzer qui, depuis 2004, utilise des documents de surveillance produits par le gouvernement américain dans le cadre de la guerre contre le terrorisme et qui ont été rendus publics. Ses Dust Paintings adoptent le style de tableaux abstraits, mais dans les grands aplats et formes géométriques de couleurs sombres se glissent des extraits de ces archives. Des mots comme «secret » ou «groupe terroriste», des phrases entières ou encore des dates donnent une idée de la source utilisée, mais jamais de manière explicite. L'impulsion de l'anarchive est ici comparable à celle qu'on trouve chez Cornelia Schleime. Dans les deux cas, les artistes ne divulguent pas toutes les informations afin de donner au spectateur un aperçu limité qui, en retour, laisse entrevoir l'ampleur des archives d'origine. En surface, les tableaux de Holzer semblent proposer une vision abstraite de la crise contemporaine, ainsi transformée en données cryptées. Mais à y regarder de plus près, on observe une texture bien présente à la surface de l'œuvre. Ce qui semblait plat prend tout à coup du relief, ce changement haptique révélant une qualité viscérale et inattendue qui rappelle les corps affectés par ces opérations secrètes (Sheets 2014). Le spectateur se trouve dans un premier temps renvoyé à lui-même par la dimension contemplative du langage abstrait de l'œuvre; mais ce mouvement d'intériorité est dans un second temps renversé par une tension qui, à la surface de la toile, lui intime d'ouvrir l'œil. L'expérience du regard se transforme en acte de surveillance. Le spectateur se retrouve adroitement impliqué dans des opérations gouvernementales qui prennent la forme déguisée de données technocratiques ou de messages revendiquant la sécurité nationale. 
La Série Stasi est beaucoup moins subtile. Même si les spectateurs doivent savoir l'allemand (ou disposer d'une traduction) pour comprendre les textes, le message de chaque planche se donne clairement à voir. Cet exhibitionnisme peut être interprété comme un antidote au secret et à l'hypocrisie dont l'artiste fut victime pendant de nombreuses années. Ses textes sont à l'image de ceux que Holzer enfouit dans ses tableaux. C'est elle que I'on a regardée. Aucun secret n'entoure plus cette opération de surveillance. La RDA est morte. Pourtant, l'usage parodique et polyvalent que Schleime fait de son dossier n'a rien de mélancolique. Contrairement à ce qu'on pourrait attendre, elle refuse d'être affectée par ce retour sur son expérience de la surveillance - sans parler de la révélation de sa trahison par un ami proche -, dix ans après son départ. En essayant de prendre de la distance par rapport à la biographie de ses années Stasi, Schleime travaille hors cadre, elle se montre même hermétique: en un mot, elle est anomique. Foster pourrait qualifier cette attitude d'utopique, «car pourquoi vouloir se connecter aussi intensément si les choses ne semblaient pas d'abord si effroyablement déconnectées entre elles? » (Foster 2004: 22). Cependant, Foster remarque que cet usage idéaliste de l'archive s'avère dans une certaine mesure productif: "Ce geste qui consiste à transformer les "sites d'excavation" en "sites de construction" est bienvenu pour une autre raison encore: cela indique une prise de distance avec une culture mélancolique dans laquelle I'historique est le plus souvent associé au traumatique. " (Ibid.) Cornelia Schleime résiste à la possibilité de succomber à sa propre histoire en produisant de nouveaux matériaux à partir des documents résultant de son oppression. II ne s'agit pas là d'une récupération de l'histoire, même si se joue une certaine forme de restauration.

L'avantage pour Cornelia Schleime est qu'elle réalise la performance en 1993, depuis son présent. Cet écart temporel lui offre une distance à la fois matérielle et émotionnelle avec les textes documentant son oppression. Le temps des archives est double: les années 1980 et l'année 1993. Ces deux temporalités renvoient également à des origines matérielles distinctes. Un premier état correspond aux archives d'origine restées secrètes, augmentées ensuite par Cornelia Schleime qui s'autorise à les mettre pleinement au jour. En tant qu'œuvre d'art, la Série Stasi résiste à une chronologie simpliste. Artefact culturel témoignant du temps d'une nation aujourd'hui disparue, elle démontre l'instabilité du concept même de nation, de son langage et de ses armes de pouvoir, des critères à l'aune desquels ce pays évaluait le caractère normal ou anormal de tel ou tel comportement pour asseoir sa souveraineté. La force de cette œuvre réside alors peutêtre dans sa dimension préfigurative. Un jour viendra-t-il où, nous aussi, nous pourrons traiter avec le même humour les dossiers de surveillance de la NSA ${ }^{10}$ ? Ou bien le format analogique des archives de la Stasi, ainsi que l'implication d'informateurs dans la rédaction de ces dossiers, les rendraient-ils trop humains, trop concrets pour qu'une même approche soit possible? En résumé, peut-être le projet de Cornelia Schleime représentet-il plus généralement l'expérience matérielle du temps qui passe, ou tout au moins la croyance selon laquelle il est possible d'observer le temps et de le mesurer avec des données matérielles. Le titre Dans l'attente de la poursuite d'une bonne collaboration, Nr. 7284/85 implique en effet une conception très concrète de l'avenir. La Série Stasi reproduit les différentes
10. La National Security Agency est un organe du département de la Défense américain, notamment en charge du renseignement. $[\mathrm{NdT}]$ 





matérialités de ces archives: les pages d'origine, les impressions, les sérigraphies, les photographies.

Certaines parties de la série inscrivent la performativité du projet dans cette matérialité (fig. 10). Dans une photographie en noir et blanc, l'artiste est vêtue comme une femme au foyer. Elle a noué un fichu sur sa tête et porte un long tablier imprimé. Le regard fixé sur l'objectif, elle incline son verre vers le spectateur pour porter un toast. La scène a lieu en présence d'une idole silencieuse derrière elle. II s'agit d'Erich Honecker, le leader du Parti socialiste unifié d'Allemagne. Sur son portrait, copie exacte de l'image officielle affichée dans toute la RDA, sont fixées les aiguilles d'une horloge. Sous ce double portrait (Schleime/Honecker) apparaissent quatre lignes de texte: «L'enquête étant terminée, on peut conclure que ses comportements sociaux et politiques expliquent ses motivations pour émigrer et qu'elle refuse complètement de se conformer à la société socialiste. " Cornelia Schleime ne s'arrête pas à ce constat: elle met en image son refus et transforme son ennemi en décor pop. 
Bibliographie

\section{Bathrick, David}

1995, The Powers of Speech: The Politics of Culture in the GDR. Lincoln/Londres, University of Nebraska Press.

\section{Bethke, Eckart}

1985 «Trabbis, Ladas und Genossen ", in Werner Filmer et Heribert Schwan (dir.), Alltag im anderen Deutschland. Düsseldorf/ Vienne, Econ Verlag : 57-64.

\section{Boyer, Dominic}

2001 «Foucault in the Bush: The Social Life of Post-Structuralist Theory in East Berlin's Prenzlauer Berg ", Ethnos (Estoclomo) 66: 207-236.

\section{Butler, Judith}

2009 Ces corps qui comptent: de la matérialité et des limites discursives du "sexe", trad. de l'anglais par Charlotte Nordmann. Paris, Éditions Amsterdam.

\section{Calle, Sophie}

1993 "Sophie Calle in conversation with Bice Curiger ", in Adrian Searle (éd.), Talking Art 1. Londres, Institute of Contemporary Arts : 29-42.

\section{Derrida, Jacques}

$1995 \mathrm{Mal}$ d'archive:

une impression freudienne.

Paris, Galilée.

\section{Dölling, Irene}

1990 «Frauen- und Männerbilder. Eine Analyse von Fotos in DDRZeitschriften ", Feministische Studien 8 (1) : 35-49.

\section{Fenemore, Mark}

2007 Sex, Thugs, and Rock ' $N$ ' Roll: Teenage Rebels in Cold-War East Germany. New York/Oxford, Berghahn Books.

La femme de leurs rêves. Par Sara Blaylock

\section{Ferree, Myra Marx}

2012 Varieties of Feminism:

German Politics on Global

Perspective. Stanford, Stanford University Press.

\section{Foster, Hal}

2004 "An Archival Impulse",

October 110 (1) : 3-22.

\section{Foucault, Michel}

1969 L'Archéologie du savoir. Paris, Gallimard.

\section{Fulbrook, Mary}

1995 Anatomy of a Dictatorship: Inside the GDR, 1949-1989. New York, Oxford University Press.

\section{Gieseke, Jens}

2014 The History of the Stasi: East Germany's Secret Police 1945-1990, trad. de l'allemand par David Burnett. New York/Oxford, Berghahn Books.

\section{Gillen, Eckhart}

2002 "Der Osten ist grau: Cornelia Schleime ", in Eugen Blume et Bernd Lindner (éds.), Wahnzimmer. Klopfzeichen. Kunst und Kultur der 80er Jahre in Deutschland, catalogue d'exposition (Leipzig, Museum der bildenden Künste / Essen Museum Folkwang). Leipzig, Faber \& Faber : 158-163.

\section{González, Jennifer and Gómez-Peña, Guillermo}

2009 «Pose and Poseur:

The Radical Politics of Guillermo Gómez-Peña's PhotoPerformances ", in Ilona Katzew et Susan Deans-Smith (dir.), Race and Classification: The Case of Mexican America. Stanford, Stanford University Press : 236-263.

\section{Jones, Amelia}

2005 «Roberta Breitmore Lives On ", in Meredith Tromble (dir.), The Art and Films of Lynn Hershman Leeson: Secret Agents, Private I. Berkeley/Los Angeles, University of California Press: 104-111.

\section{Kaiser, Paul}

2009 «Das Gift der Diktatur. Die Dresdner Gegenkultur im Visier der Staatssicherheit ", in Frank Eckhardt et Paul Kaiser (dir.), Ohne uns! Kunst \& alternative Kultur in Dresden vor und nach ' 89. Dresde, Efau Verlag: 108-119.

\section{Kaiser, Paul et Petzold,} Claudia

1997 Boheme und Diktatur in der DDR: Gruppen, Konflikte, Quartiere 1970-1989, catalogue d'exposition (Berlin, Deutschen Historischen Museums).

Berlin, Fannei \& Walz Verlag.

\section{Maier-Schaeffer,} Francine

2015 «Le spectre de I'histoire. Weit fort (2008) de Cornelia Schleime, un "Wenderoman seconde génération"? ", Études germaniques 278: 337-355.

\section{Müller-Enbergs, Helmut}

2008 Die inoffiziellen Mitarbeiter Berlin, Der Bundesbeauftragte für die Unterlagen des Staatssicherheitsdienstes der ehemaligen Deutschen Demokratischen Republik, Abteilung Bildung und Forschung.

\section{Mulvey, Laura}

1991 «A Phantasmagoria of the Female Body: The Work of Cindy Sherman ", New Left Review 188 : 136-150.

\section{Pejić, Bojana (dir.)}

2009 Gender Check: Femininity and Masculinity in the Art of Eastern Europe, catalogue d'exposition Vienne, Museum Moderner Kunst Stiftung Ludwig/ Zachęta National Gallery of Art). Cologne, Buchhandlung Walther König.

\section{Schleime, Cornelia}

2008 Weit for. Regensburg Hoffmann und Campe.

2010 In der Liebe und in der Kunst weiß ich genau, was ich nicht will. Bielefeld/Leipzig/Berlin, Kerber Verlag.

2011 " lch sagte, ich trete in den Hungerstreik ", interview par Herlinde Koelbl, ZEITmagazin 35 (25 août 2011) [en ligne], disponible sur: http://www.zeit.de/2011/35/ Rettung-Cornelia-Schleime (consulté le 29 mars 2016).

\section{Sheets, Hilarie M.,}

2014 « Jenny Holzer. New York at Cheim \& Read ", Art in America (4 novembre 2014) [en ligne], disponible sur: http://www. artinamericamagazine.com/ reviews/jenny-holzer-1/, (consulté le 17 mars 2016). 

it vom 24.10 .83 (Blatt $74-75$ des Hat.) hinausveise bekannt geworden, da

Films is berrest.

Hendel, Annekatrin

2014 Anderson. Allemagne,

Salzgeber, 1 h 35 min (DVD, 2015).

Krusenstjerna, Fredrik (von) et Cederberg, Björn 1994 Betrayal. Suède, Stockholm, Charon Film, 58 min, VHS.

page 18 et ci-contre Cornelia Schleime, Bis auf weitere gute Zusammenarbeit, Nr. 7284/85, série en 15 parties, 1993, photographie sérigraphiée $100 \times 70 \mathrm{~cm}$ () Cornelia Schleime avec l'aimable autorisation de la galerie Michael Schultz, Berlin.
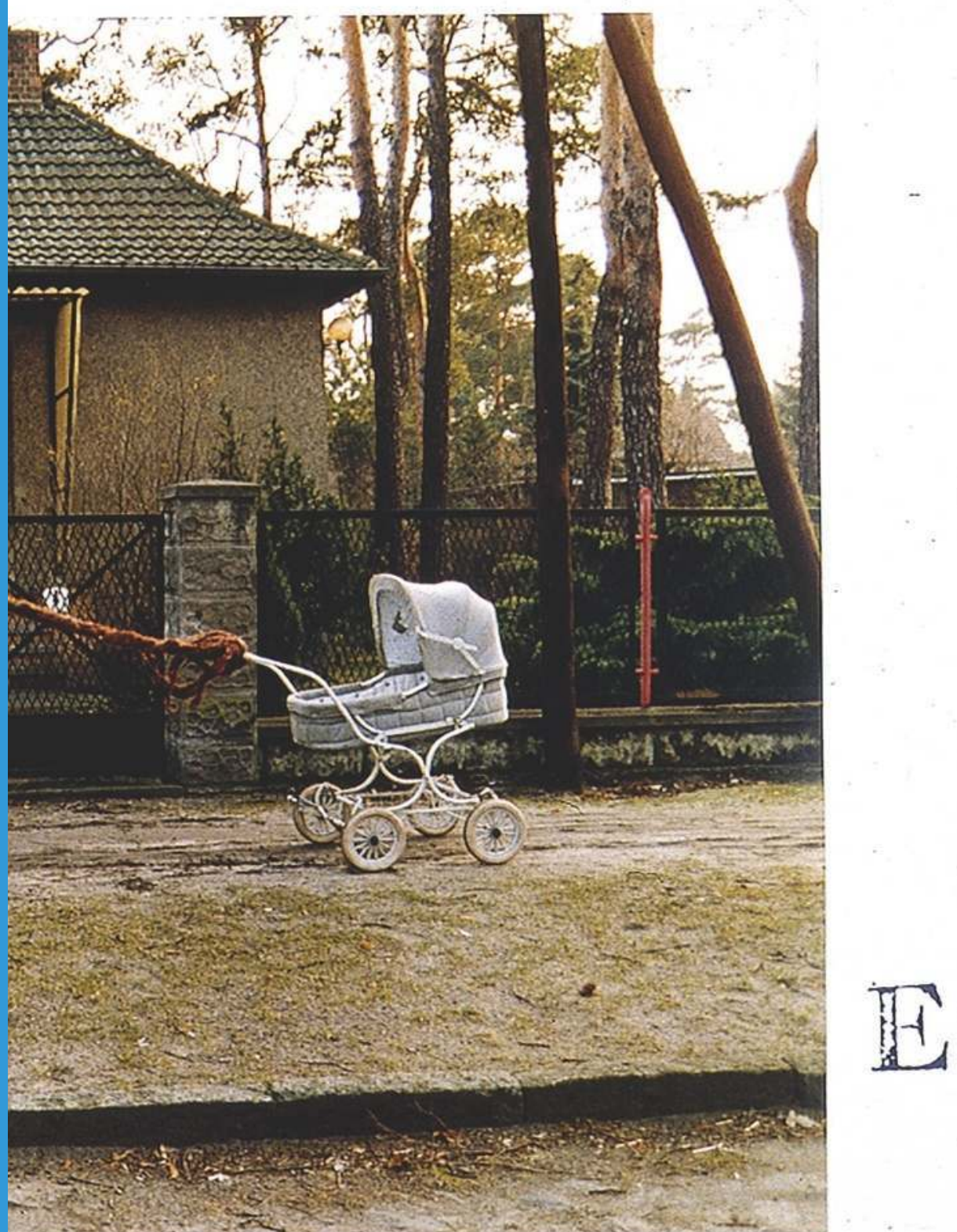

1

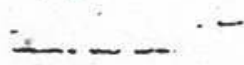

